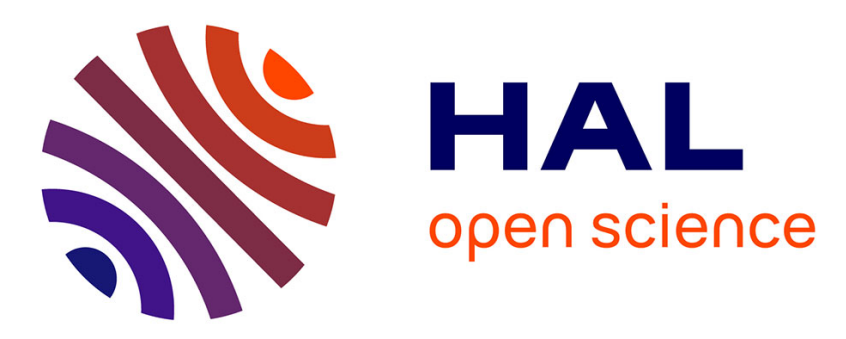

\title{
Investigation of sheet-flow processes based on novel acoustic high-resolution velocity and concentration measurements
}

Thibaud Revil-Baudard, Julien Chauchat, David Hurther, Pierre-Alain

Barraud

\section{To cite this version:}

Thibaud Revil-Baudard, Julien Chauchat, David Hurther, Pierre-Alain Barraud. Investigation of sheet-flow processes based on novel acoustic high-resolution velocity and concentration measurements. Journal of Fluid Mechanics Digital Archive, 2015, 767, pp.1-30. 10.1017/jfm.2015.23 . hal-01122373

\section{HAL Id: hal-01122373 \\ https://hal.science/hal-01122373}

Submitted on 5 Mar 2015

HAL is a multi-disciplinary open access archive for the deposit and dissemination of scientific research documents, whether they are published or not. The documents may come from teaching and research institutions in France or abroad, or from public or private research centers.
L'archive ouverte pluridisciplinaire HAL, est destinée au dépôt et à la diffusion de documents scientifiques de niveau recherche, publiés ou non, émanant des établissements d'enseignement et de recherche français ou étrangers, des laboratoires publics ou privés. 


\title{
Investigation of sheet-flow processes based on novel acoustic high-resolution velocity and concentration measurements.
}

\author{
Thibaud Revil-Baudard ${ }^{1} \dagger$, Julien Chauchat ${ }^{1}$, David Hurther ${ }^{1}$ and \\ Pierre-Alain Barraud ${ }^{1} \ddagger$, \\ ${ }^{1}$ LEGI, Grenoble University, Domaine Universitaire, BP 53 , 38041 Grenoble Cedex 9, France
}

(Received ?; revised ?; accepted ?. - To be entered by editorial office)

A new dataset of uniform and steady sheet flow experiments is presented in this paper. An Acoustic Concentration and Velocity Profiler (ACVP) is used to measure time-resolved profiles of collocated $2 \mathrm{C}$ velocity $(u, w)$ and sediment concentration and to measure the time evolution of the bed interface position. Ensemble averaging over eleven similar experiment realisations is done to evaluate the mean profiles of streamwise velocity, concentration, sediment flux and Reynolds shear stress. The repeatability, stationarity and uniformity of the flow are carefully checked for a Shields number $\theta \approx 0.5$ and a suspension number of $S=1.1$. The mean profile analysis allows to separate the flow into two distinct layers: a suspension layer dominated by turbulence and a bed layer dominated by granular interactions. The bed layer can be further subdivided into a frictional layer capped by a collisional layer. In the suspension layer, the mixing length profile is linear with a strongly reduced von Karman parameter equal to 0.225. The Schmidt number is found to be constant in this region with a mean value of $\sigma_{s}=0.44$. The present results are then interpreted in terms of existing modelling approaches and the underlying assumptions are discussed. In particular, the well-known Rouse profile is shown to predict the concentration profile adequately in the suspension layer provided that all the required parameters can be evaluated separately. However, the strong intermittency of the flow observed in the bed layer under the impact of turbulent large-scale coherent flow structures suggests the limitations of averaged steady two-phase flow models.

Key words:

\section{Introduction}

The sheet flow regime of sediment transport occurs when the fluid flow is sufficiently strong to mobilise a thick and dense layer of particles on the top of a sediment bed. The present paper is focused on uniform, steady and turbulent sheet flows of well-sorted particles in which the low value of the slope allows to neglect the body force acting on the particles. In such conditions, both granular interactions and turbulent processes are the key mechanisms in momentum and particle diffusion (e.g. Bagnold 1956; Jenkins \& Hanes 1998). The Shields number $\theta$ and the suspension number $S$ are the two dimensionless numbers that control sediment transport in this regime (Sumer et al. 1996). The Shields

$\dagger$ Email address for correspondence: thibaud.revil-baudard@legi.grenoble-inp.fr

$\ddagger$ Current affiliation: TIMC-IMAG, GMCAO team,In3S, Pavillon Taillefer, Rond-Point de la Croix de Vie, La Tronche, France 
number is defined as the ratio between the force exerted by the fluid on a particle at the bed and the apparent weight of a single particle: $\theta=\rho_{f} u_{*}^{2} /\left(\rho_{p}-\rho_{f}\right) g d_{p}$ where $\rho_{f}$ and $\rho_{p}$ are the density of the fluid and the particles respectively, $u_{*}$ is the friction velocity, $g$ the acceleration of gravity and $d_{p}$ the particle diameter. In the literature, the transition from the bedforms regime to the sheet flow regime occurs at a Shields number around 0.5. Above this value, the bed-load layer thickness and the associated solid load increase with the Shields number (e.g. Einstein 1950; Yalin 1977). The suspension number, $S=w_{s} / u_{*}$, characterises the competition between the particle inertia represented by its settling velocity $\left(w_{s}\right)$ and the magnitude of the turbulent velocity fluctuations represented by the friction velocity $\left(u_{*}\right)$. Following Sumer et al. (1996), for $S<0.8$ the sheet-flow regime is in the suspension mode in which the suspended-load is greater than the bedload. For $S>1$, the sheet-flow regime is in the no-suspension mode in which the bed-load dominates over the suspension one.

The challenges in research on the sheet flow regime reside currently in two complementary aspects. The first one lies in the modelling of the horizontal and vertical momentum transfer mechanisms for both fluid and solid phases. The second one concerns the realisation of controlled sheet flow experiments providing reliable high-resolution data. At present, only few such datasets exist in the literature and this strongly limits our ability to investigate the validity of sheet flow models.

The vertical profile of shear stress (i.e. along the flow normal direction) from the freesurface down to the non-moving bed can be derived from the horizontal (i.e. streamwise) momentum balance. Under uniform flow conditions, the total shear stress $\tau$ is linear with $z$, the vertical coordinate, and at any given position it balances the weight of the water column above, projected on the streamwise direction $x$. Both the fluid and the granular phase possibly contribute to the total shear stress. In the dilute suspension (usually taken as the flow domain where the volumetric concentration $\phi$ is lower than 0.08 , Hsu et al. 2004) the total shear stress is balanced by the turbulent Reynolds shear stress which transfers streamwise momentum downward from high velocity layers to reduced velocity layers. Within the mobile sediment layer, momentum can be transferred vertically by turbulent fluctuations and/or collisional and frictional interactions between grains. At the bed interface, a plastic threshold imposes a zero velocity shear rate where the friction between grains exactly balances the total shear stress (Coulomb yield criterion, Hanes \& Inman 1985). The vertical profile of particle concentration can be obtained from the vertical momentum balance in which gravity is mainly balanced by two mechanisms: a turbulent dispersion term arising from the effect of turbulent velocity fluctuations on the drag force (e.g. Chauchat \& Guillou 2008) and a dispersive force induced by granular interactions (Bagnold 1954; Wilson 1989; Jenkins \& Hanes 1998). This last force originates from collisions and enduring contacts between particles and vanishes at low concentration $(\phi \lesssim 0.08)$ for which particles are fully suspended by the fluid turbulence. Around this limit value of concentration it is likely that a competition between both mechanisms occurs.

Several attempts have been made to model the above mentioned granular interactions in sheet flow regime. In his pioneering work, Bagnold (1954) has proposed a formulation of the granular shear stress proportional to the square of the velocity shear rate and to the square of the particle diameter (inertial regime). He also suggested that the particulate pressure is proportional to the shear stress. More recently, several authors have used the kinetic theory of granular flows to account for the granular contribution to the shear stress (Jenkins \& Hanes 1998; Hsu et al. 2004; Berzi 2011, amongst others). In this theory, an additional energy equation has to be solved for the granular temperature which represents the intensity of the particle velocity fluctuations. This variable is used to eval- 
uate the granular shear and normal stresses. During the last decade a phenomenological rheology, $\mu(I) / \phi(I)$, has been proposed for dry granular flows (GDR midi 2004; Forterre \& Pouliquen 2008) in which the inertial number $I$, representing the competition between the timescale of rearrangement to the timescale of deformation of the granular media, controls the shear to normal stress ratio $\mu$ and the particle concentration $\phi$ (Andreotti et al. 2013). This rheology has been used with some success to model laminar bed-load (Ouriemi et al. 2009) and turbulent sheet flow (Revil-Baudard \& Chauchat 2013).

Concerning the turbulent contribution to the total shear stress, it has been observed that the presence of particles can significantly modify the turbulence compared with a clear water turbulent shear boundary layer (Best et al. 1997; Muste et al. 2005). In order to account for this effect, several authors have used a mixing length approach with a correction depending on (a) the density stratification rate (e.g. Jenkins \& Hanes 1998), (b) the local concentration (Pasini \& Jenkins 2005, for example) or (c) the integral of the concentration profile (Revil-Baudard \& Chauchat 2013). Hsu et al. (2004) have employed a $k-\epsilon$ model with a transfer term related to the particle inertia to account for turbulence modulation induced by the presence of particles. However this turbulence modification remains an open question widely discussed in the research community (Castro-Orgaz et al. 2012). The concentration profile in the dilute region of the flow is usually modelled by the so-called Rouse profile. It is based on a balance between the downward settling flux and the upward turbulent dispersion flux. This last term is classically modelled by a Fickian diffusion law as the product of the sediment diffusivity times the local vertical gradient of concentration (Graf \& Cellino 2002). The sediment diffusivity is related to the turbulent eddy viscosity divided by the Schmidt number $\left(\sigma_{s}\right)$ which is usually tuned to fit experimental measurements.

Laboratory experiments for the study of the vertical structure of turbulent 2D mean sheet flows are very rare so far. The main reasons for this are the difficulties of measuring both the velocity and the sediment concentration in the same sample volume under such extreme concentration conditions and with a spatio-temporal resolution sufficiently high to resolve turbulent eddies down to the particle scale. Furthermore, the importance of the Reynolds shear stress in the momentum balance across the active sheet flow layer imposes quasi-instantaneous two-component $(2 \mathrm{C})$ velocity measurement performances. Few flow measurement systems are adapted to such challenging flow conditions. Experiments were mainly conducted in pipe flows by Daniel (1965); Wilson (1966); Nnadi \& Wilson (1992); Pugh \& Wilson (1999). These studies examined the time-averaged volumetric concentration and streamwise velocity $(u)$ using a point-wise gamma-ray technique and a point-wise conductivity probe, respectively. Sumer et al. (1996) carried out sheet flow experiments in an open-channel flume (with and without a rigid lid at the top) using point-wise capacity probes for the concentration measurements and a Pitot-tube for the local streamwise velocity measurements. More recently, Cowen et al. (2010) have developed a Boroscopic technique to measure the streamwise velocity profile inside the dense moving bed layer. However, a single velocity profile corresponding to one flow configuration has been published so far. All these experiments used intrusive point wise techniques subjected to local flow perturbations and providing only non-colocated one-component (1C) velocity and concentration time series. Consequently, the analysis of these data was restricted to the interpretation of mean profiles of independently measured quantities with no direct quantification of the co-variances such as the Reynolds shear stress or the turbulent sediment fluxes. More recently, Armanini et al. (2005); Spinewine et al. (2011) and Capart \& Fraccarollo (2011) have developed a video technique to get instantaneous $2 \mathrm{C}$ particle velocity and mean concentration profiles in the near-wall region. To the best of our knowledge, this dataset is currently the only one which provides both particle 
velocity and concentration profiles under sheet flow conditions in an open channel flow. The fact that velocity and concentration measurements are not collocated neither synchronised and that they were measured at the sidewall constitute the main restrictions of this dataset.

In the present paper, a new sheet flow experiment carried out in an open-channel is presented. A multi-frequency Acoustic Concentration Velocity Profiler (ACVP, Hurther et al. 2011; Naqshband et al. 2014) is used to provide non-intrusive vertical profiles across the suspension and the sheet flow layers of co-located $2 \mathrm{C}$ velocities and particle concentration. The mean profiles are analysed to discuss the physical mechanisms responsible for the momentum and concentration fluxes across the entire water column and to assess different available modelling approaches. The paper is organised as follows: in section 2, the experimental setup and the data processing of the acoustic measurements are described. In section 3, the mean profiles of streamwise velocity, concentration, turbulent shear stress and sediment flux are presented. The vertical structure of the flow is outlined from momentum and concentration diffusivity profiles and further analysed based on mixing length, Stokes and Inertial numbers. Then, the measurements are compared with different constitutive laws to discuss the modelling perspectives of this new dataset. In the section 4 the obtained results are discussed and in section 5, the main conclusions of this paper are summarised.

\section{Experimental set up and flow conditions}

In this section, we first present the experimental setup composed of the tilting flume and the measurement equipment. Secondly, the experimental protocol and methodology are described. Finally, the data processing method and the corresponding mean flow data are analysed to verify (a) the establishment of 2D mean flow conditions as a steady uniform sheet flow and (b) the validity of the sediment transport measurements provided by the ACVP .

\subsection{Experimental facility}

The LEGI/ENSE3 tilting flume is $L=10 \mathrm{~m}$ long and $W=0.35 \mathrm{~m}$ wide with a bed slope set to a value of $S_{0}=0.005$. A $3 \mathrm{~m}$ long by $11 \mathrm{~cm}$ high sediment reservoir is installed in the channel bed at $2 \mathrm{~m}$ before the channel outlet (see figure 1). Elsewhere the flume bed is covered by glued particles to enhance the bed roughness and facilitate the full development of the boundary layer before the test section. The sediments are irregularly shaped (PMMA, density $\rho_{p}=1192 \mathrm{~kg} / \mathrm{m}^{3}$ ) with a tangent of the angle of repose measured at $\mu_{s}=0.7$ in dry conditions. The particle size distribution is wellsorted and the particle diameters are in the range $d_{p} \in 2.5-3.5 \mathrm{~mm}$ (table 1). The mean settling velocity is equal to $w_{s}=5.59 \pm 1.1 \mathrm{~cm} / \mathrm{s}$ which was determined experimentally from settling tests in a still water tank. The flume is equipped with an acoustic limnimeter mounted on a moveable trolley for the measurement of the water level time series at a rate of $200 \mathrm{~Hz}$ in the test section. These water level measurements are synchronised with the data acquisitions of the Acoustic Concentration and Velocity Profiler (ACVP). The ACVP is a 1D2C system measuring the co-located streamwise and vertical velocity components as well as the particle volumetric concentration over a vertical profile of 25 $\mathrm{cm}$ extending from the bed interface to the top of the free-surface. The originality of this acoustic flow instrumentation lies in the combination of the multi-bistatic ADVP technology (Acoustic Doppler Velocity Profiler Hurther \& Lemmin 2001, 2008; Mignot et al. 2009) with the multi-frequency ABS technology (Acoustic Basckscattering System, Thorne \& Hanes 2002; Thorne et al. 2011; Thorne \& Hurther 2014) into a single ACVP 
TABLE 1. Sediment and flow properties.

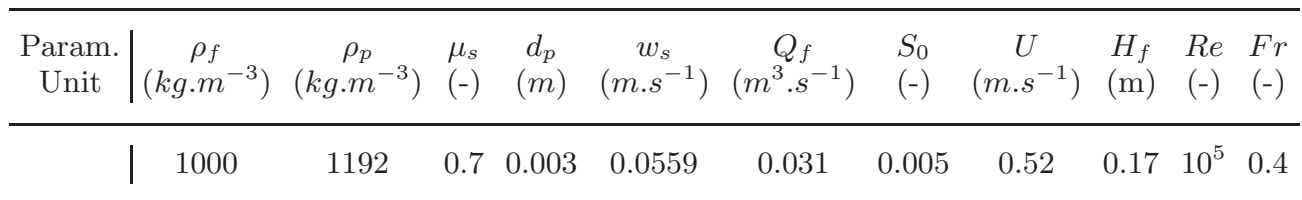
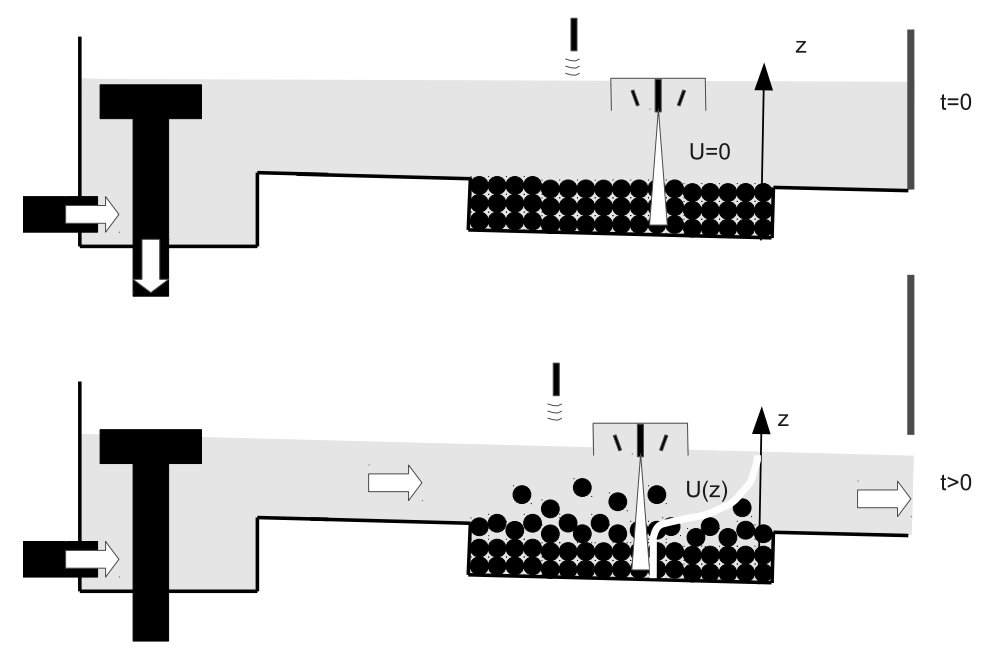

FIGURE 1. Sketch of experimental set up in its initial (a) and running (b) states.

system as proposed by Hurther et al. (2011). Recently, the profiling of velocity and sediment transport quantities across both the suspension and the bed layers has provided new insights into a variety of sediment transport processes (Hurther \& Thorne 2011; Chassagneux \& Hurther 2014; Naqshband et al. 2014). The same methodology and data validation technique is applied herein as discussed below. The spatio-temporal resolution of the measurements is equal to $\Delta z=3 \mathrm{~mm}$ along the vertical direction and $1 / 78$ $\mathrm{s}$, respectively. The time rate of the concentration measurement is set to $4.9 \mathrm{~Hz}$ to guarantee a statistical bias lower than $15 \%$ as discussed in Thorne \& Hurther (2014). The Acoustic Bed Interface Tracking (ABIT) method proposed by Hurther \& Thorne (2011) is used here for the localisation of the bed interface at a time rate of $7.8 \mathrm{~Hz}$. This bed localisation technique allows to reduce Doppler noise effects in the mean velocity and sediment transport profiles by forcing the instantaneous Doppler velocities to zero below the detected bed level position. Flow intrusiveness of the ACVP sensors is minimised by placing the system into a vacuum box. The lower end of this box is slightly below the free-surface level as illustrated in figure 1. Despite the fact that the box generates a roughly $1 \mathrm{~cm}$ thick mixing layer in the upper part of the water column, the vacuum box guarantees a negligible impact of the instrument on the sediment bed. Moreover, it provides velocity measurements over the entire shear boundary layer height as shown below.

\subsection{Experimental protocol and flow properties}

The particles transported during the experiment are not recirculated. This induces bed erosion during the runs which requires a particular attention to fulfil steady uniform flow 
conditions as discussed below. In order to minimise the transient period associated with the set up of the targeted flow discharge, the flume is first filled slowly with no sediment movement on the horizontally levelled particle bed until the still water level has reached a value just above the aimed normal flow depth. At this stage, the sluice gate at the flume exit is still closed and a circular shaped weir located in the upstream reservoir evacuates the entire water discharge as illustrated in the upper sketch of figure 1. The sluice gate is suddenly opened provoking a rapid water level drop to inactivate the circular weir and to transfer the entire flow discharge of $Q_{f}=31 \mathrm{l} / \mathrm{s}$ into the tilting flume. After less than 30 seconds the water flow and the sheet flow layer are fully developed with a normal flow depth of $H_{f}=0.17 \mathrm{~m}$ above the bed interface and a mean bulk flow velocity of $U=0.52$ $\mathrm{m} / \mathrm{s}$. This experimental procedure is repeated for $N=11$ runs in order to increase the statistical convergence of the averaged flow quantities as shown in the next section.

The bulk Reynolds number $R e=U H_{f} / \nu_{f}$ is of the order of $10^{5}$. The Reynolds roughness number $R e_{*}=u_{*}^{\prime} k_{s} / \nu_{f}$ can roughly be estimated from the friction velocity as $u_{*}^{\prime}=\sqrt{g H_{f} S_{0}}=0.09 \mathrm{~m} / \mathrm{s}$ and the bed roughness as $k_{s}=2.5 d_{p}$. This gives Re* $=375$. The values of the two Reynolds numbers indicate that the flow is fully turbulent and fully hydraulically rough. The flow is subcritical with a Froude number $F r=U / \sqrt{g H_{f}} \approx 0.4$ where $g$ is the gravitational acceleration.

Figure (2) presents an example of the measured profiles of the streamwise velocity (a), the volumetric particle concentration (b) and the streamwise sediment flux $\pi=\phi u$ (c) for one of the 11 runs. The black solid line represents the detected bed interface. The vertical axis is the vertical coordinate made dimensionless by the particle diameter. Due to the highly turbulent flow conditions, these data have been low-pass filtered using a moving rectangular window at a cutoff frequency of $4.9 \mathrm{~Hz}$. The black solid lines with circle markers represent the vertical profiles averaged over 6 seconds and shown every 10 seconds. As can be seen from these figures, the run can be divided into a transient phase of about $30 \mathrm{~s}$ followed by a quasi-steady bed erosion phase of roughly $40 \mathrm{~s}$ long. During the transient phase, the bed is first abruptly eroded over the first $5 \mathrm{~s}$ and more slowly accreted until $t=28 \mathrm{~s}$. The corresponding streamwise velocity field shows a severe acceleration phase reaching velocities of about $1 \mathrm{~m} / \mathrm{s}$ associated with the bed erosion phase and a bulk flow deceleration period during the accretion phase. This transient phase also shows a strong vertical heterogeneity in the velocity profiles since a low velocity nearbed region appears to persist over the first $20 \mathrm{~s}$ (seen as a blue colour nearbed domain in figure 2a). This vertical flow heterogeneity disappears after $30 \mathrm{~s}$ with the establishment of typical shear flow profiles of negligibly low velocity at the bed interface position. The volumetric concentration timeseries (in $\log _{10}$ ) show the development of a suspension layer over the first $25 \mathrm{~s}$ from an initial value $\phi \approx 0$ to values in the $\phi \approx 0.001$ range. In the nearbed region the concentration reaches a maximum value $\phi^{m}=0.55$ below the bed interface position. In particular, the maximum bed concentration value follows quite perfectly the time evolution of the bed level position over the entire run. This unforced behaviour strongly supports the validity of the acoustic concentration measurements in the dense nearbed flow region. The colorplots of the sediment flux in figure $2 \mathrm{c}$ reveals high nearbed sediment flux values over the entire run. The suspension flux over the transient phase is negligibly low. This can be attributed to the negligibly low suspension concentration values since the bulk flow velocity is significantly high during this phase. In the quasisteady erosion phase, the sediment flux profiles show fairly steady shapes of the profiles relative to the bed interface position. Although not shown here, the 11 runs show a very similar spatio-temporal structure at large flow scales and with a randomly distributed short scale variability over the different runs. The repeated run behaviour allows us to apply the ensemble averaging technique described in the following section. Another 
(a)

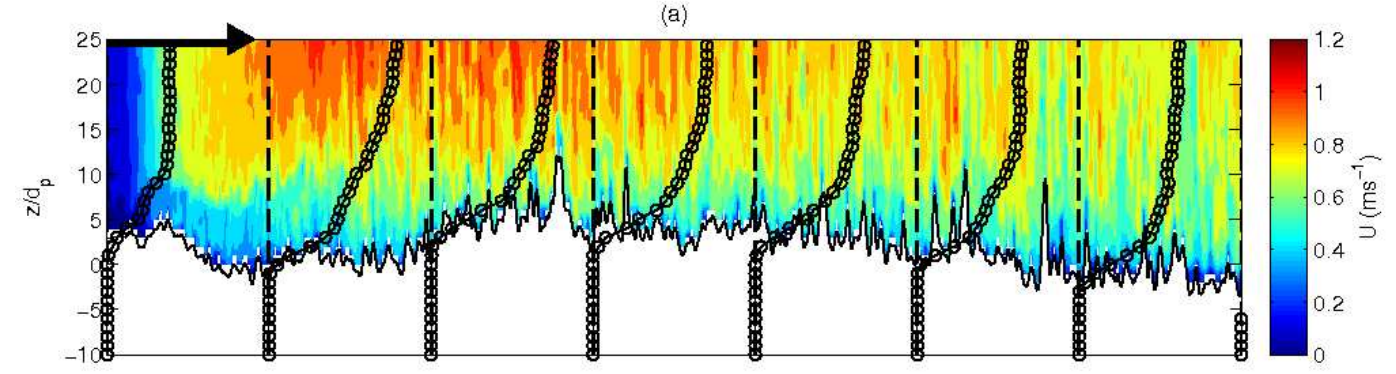

(b)

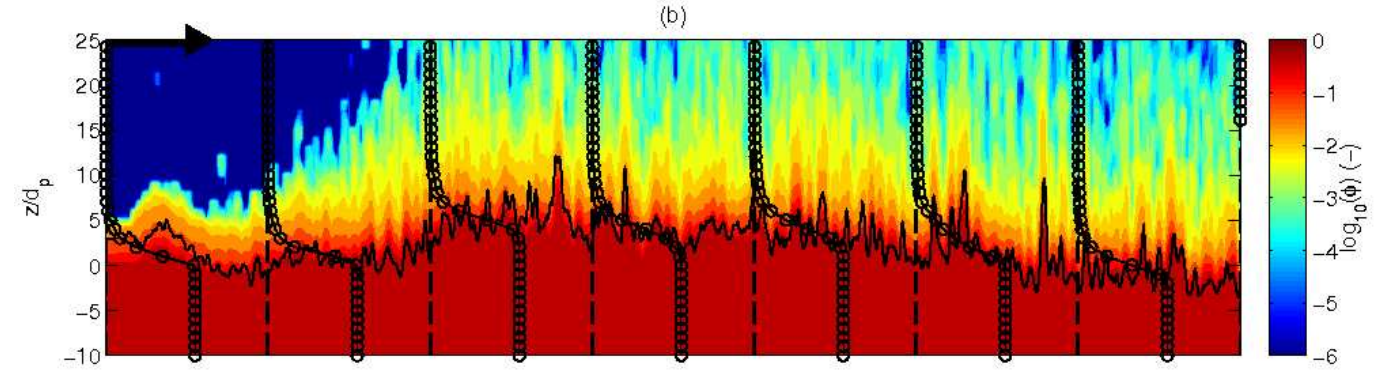

(c)

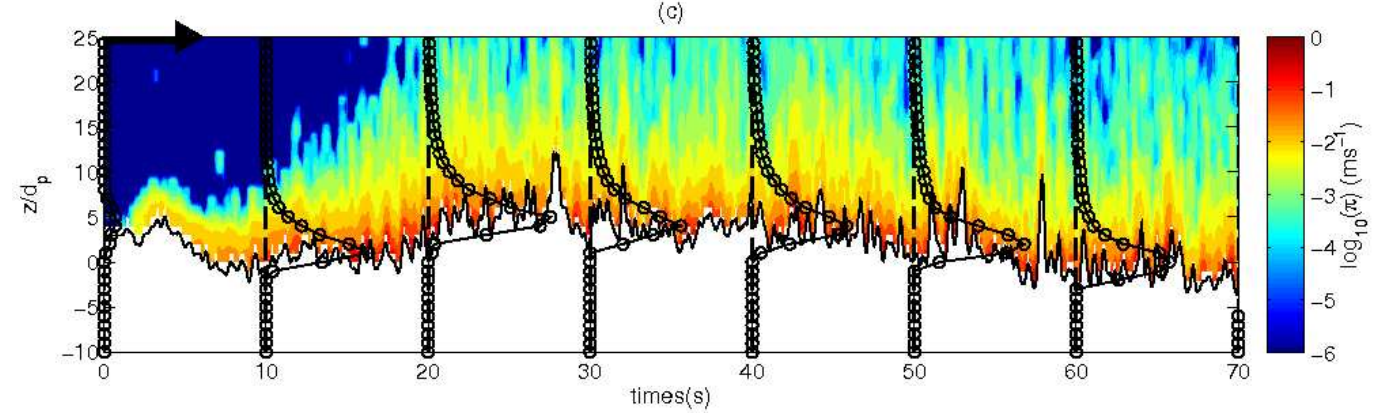

Figure 2. Colorplot of the filtered instantaneous streamwise velocity (a), concentration (b) and 2D sediment flux (c). The filtered detected bed-interface positions is presented by the solid black lines. The vertical axis is made dimensionless by the particle diameter. The curves show the vertical profiles of (a) streamwise velocity, (b) concentration and (c) sediment flux averaged over $6 \mathrm{~s}$ and represented every $10 \mathrm{~s}$. For these profiles the arrow at the top left corner of each panel corresponds to $u=1 \mathrm{~ms}^{-1}, \phi=0.55$ and $\pi=0.05 \mathrm{~ms}^{-1}$ respectively.

interesting point in figure 2 is the range of bed level variability at short timescales. It is found to reach several particle diameters. The origin of this phenomenon is addressed in the discussion of this paper. From the present analysis of the time series time it can be deduced that time averaging over the complete duration of a run is unadapted to satisfy steady uniform conditions. Therefore, a specific averaging technique has been applied as described in section 2.4 .

\subsection{Validation of acoustic sediment transport measurements}

In order to test quantitatively the validity of the acoustic sediment flux measurements, the volume of transported particles estimated acoustically has been directly compared to the volume of particles collected in two sediment traps located downstream the test section. The acoustic estimation of the total volume of transported sediment, $\mathcal{V}_{A C V P}\left(t=t_{\max }\right)$, 


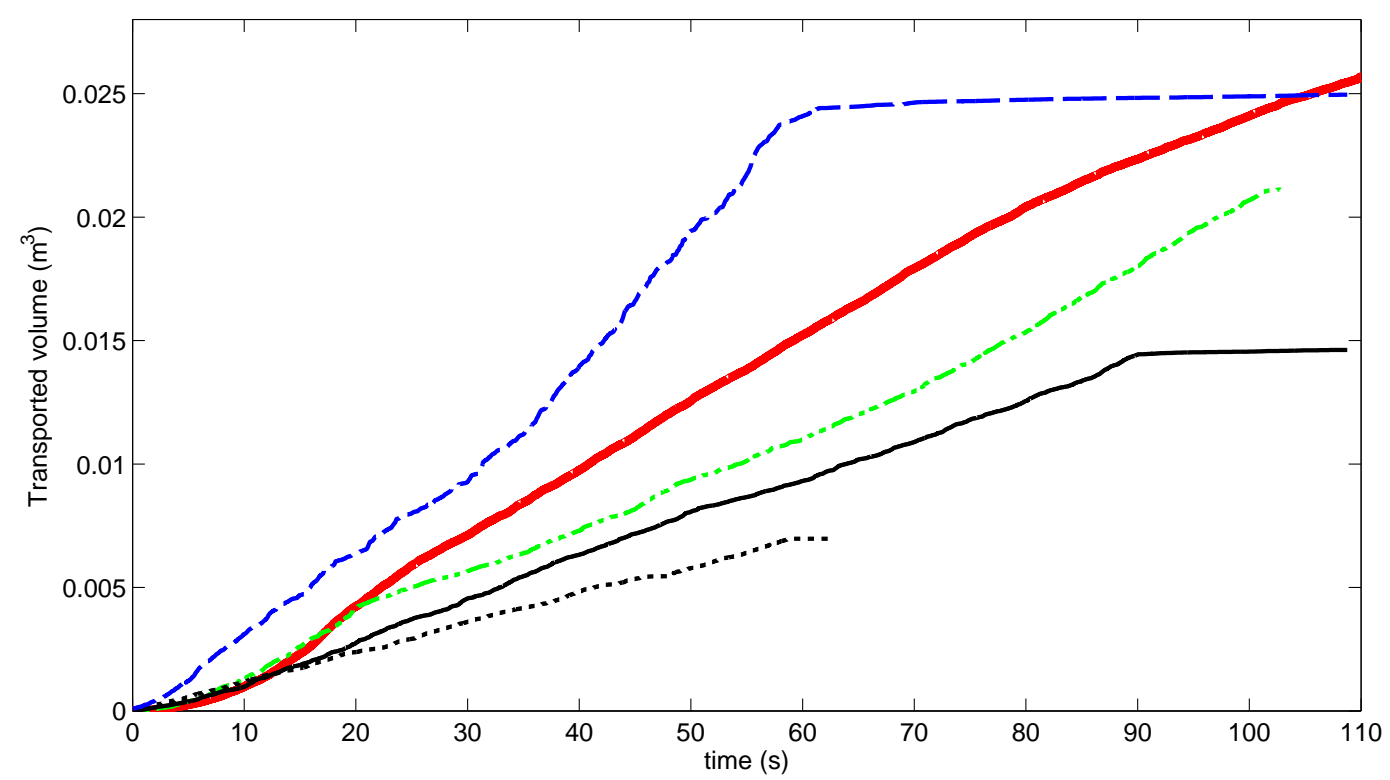

FiguRE 3. Time evolution of the volume of transported sediment (acoustic estimation) for different flow regimes, from low bed-load (...) to an intense sheet flow (- - -). The thick line corresponds to the ensemble averaged sheet flow experiment considered in this paper.

Transport mode|Low Bed-Load|Bed-Load|Sheet-Flow|Sheet-Flow + Suspension

\begin{tabular}{c|c|c|c|c}
\hline Duration (s) & 59 & 90 & 100 & 62 \\
$\mathcal{V}_{w}(\mathrm{l})$ & $6.7 \pm 0.5$ & $17.8 \pm 0.5$ & $25.7 \pm 0.5$ & $27.3 \pm 0.5$ \\
$\mathcal{V}_{A C V P}(\mathrm{l})$ & 7 & 14.5 & 21.1 & 24.5 \\
Relative error & $-5 \%$ & $18 \%$ & $18 \%$ & $10 \%$
\end{tabular}

TABLE 2. Comparison between the volume of transported sediment evaluated acoustically $\left(\mathcal{V}_{A C V P}\right)$ and by weighing $\left(\mathcal{V}_{w}\right)$.

is calculated from:

$$
\mathcal{V}_{A C V P}(t)=W \int_{0}^{t} \int_{-\infty}^{+\infty} u(t, z) \phi(t, z) \mathrm{d} z \mathrm{~d} t,
$$

where $W$ represents the flume width. The mass balance technique consists in the weighting of the dried amount of particles $\mathcal{V}_{w}$ collected after a run. The obtained mass is divided by the particle density for comparison with the acoustic estimation $\mathcal{V}_{A C V P}\left(t_{\text {max }}\right)$.

Figure 3 represents the time evolution of the transported particle volume, $\mathcal{V}_{A C V P}\left(t_{\max }\right)$. In order to carefully validate the acoustic measurement, the comparison has been done for four significantly different flow conditions (see table 2). The black dotted line corresponds to relatively low bed-load regime while the blue dashed one corresponds to an intense sheet flow. The discrepancy between the two estimations is found to be less than $18 \%$. The discrepancies for the lowest bed-load and the most intense sheet flow are $5 \%$ and $10 \%$ respectively. Since the transported volume estimated acoustically is obtained by adding up a very large number of sediment flux realisations, the obtained low relative errors support the presence of negligibly low systematic errors in the sediment flux measurements. 


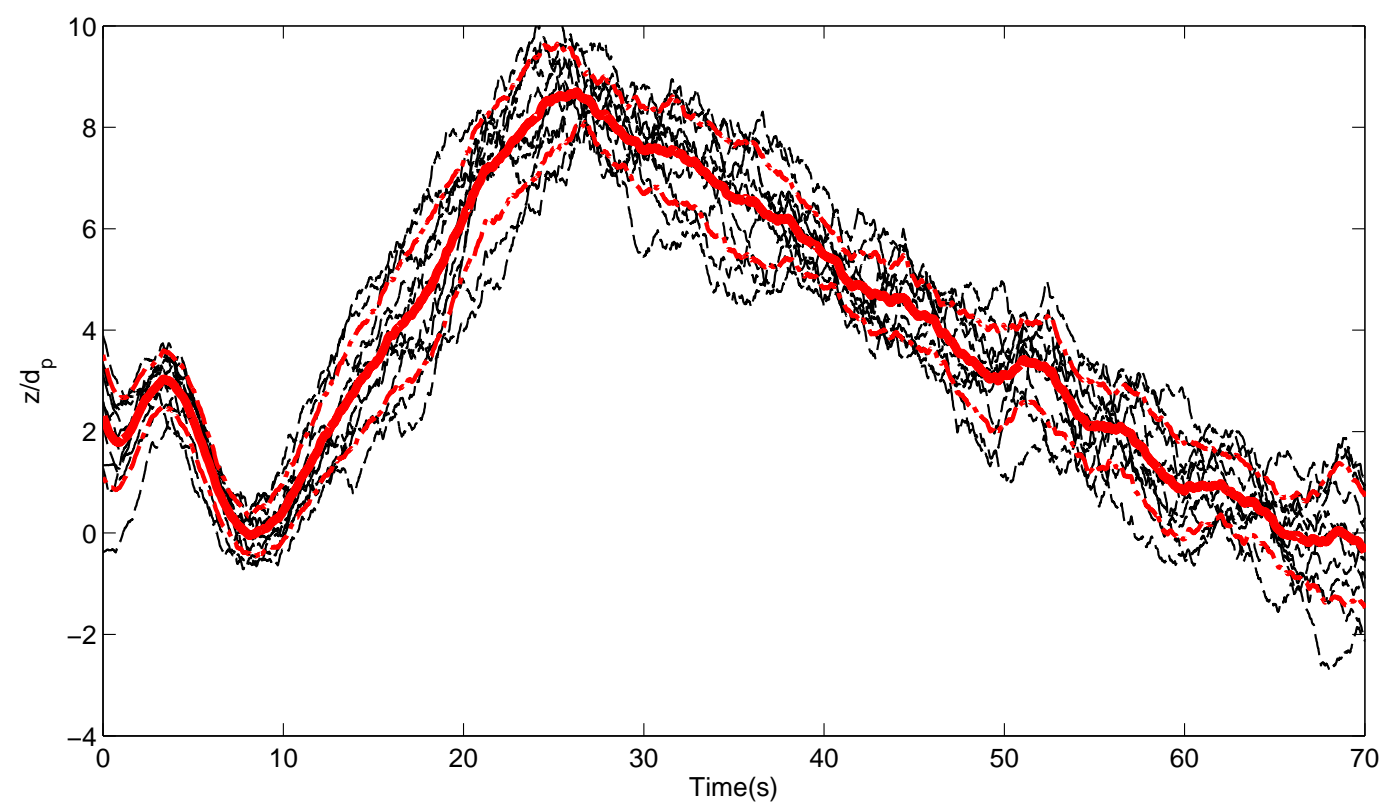

Figure 4. Time evolution of the bed interface positions filtered at $0.333 \mathrm{~Hz}$ and made dimensionless by a particle diameter for the 11 realisations (- - -). Ensemble average (-) boxed by the associated standard deviation (-.-).

Another interesting aspect is seen in the trend of the thickest curve in figure 3 which corresponds to an ensemble average of $\mathcal{V}_{A C V P}(t)$ over the 11 repeated runs. It shows a clear slope break from a high to a lower value of the sediment transport rate around $t=30 \mathrm{~s}$. This change of sediment transport rate is in very good agreement with the observations made in figure 2 on the presence of a transient phase followed by a fairly quasi-steady erosion phase. The results shown here strongly support the validity of the sediment transport measurement provided by the ACVP.

\subsection{Experiment repeatability, flow steadiness and uniformity}

In order to ensemble average the measured quantities over the eleven runs, the repeatability of the experiments is analysed herein. A representative criteria is the ensemble standard deviation associated with the 11 realisations. Figure 4 shows the time series of the bed level position for the 11 realisations of the experiment. Each realisation has been time referenced to an initial event of free-surface water drop occurring after the gate opening. The drop is set to $5 \%$ as $H_{f}(t=0)=0.95 H_{\max }$, where $H_{\max }$ is the initial water level in the upper sketch of figure 1 . In figure 4 the ensemble average of the bed level position is plotted (thick red line) and bounded by plus and minus the ensemble standard deviation (red dashed lines). It is shown that the value of the ensemble standard deviation is around one particle diameter and it is of the order of the variability in a single timeserie. This indicates the very high degree of repeatability of the experiment in terms of bed morphology evolution.

Since the bed is eroded during the experiment, the interval over which the flow can be time-averaged has to be determined. The ensemble average of the bed level timeserie (figure 4) permits the evaluation of the mean erosion rate in the quasi-steady phase $(t>30 \mathrm{~s})$ to a value of one particle diameter every 6 seconds. This time interval $(\Delta t=6$ $\mathrm{s}$ ) is sufficiently short to assume a constant bed level and long enough to be statistically 
(a)

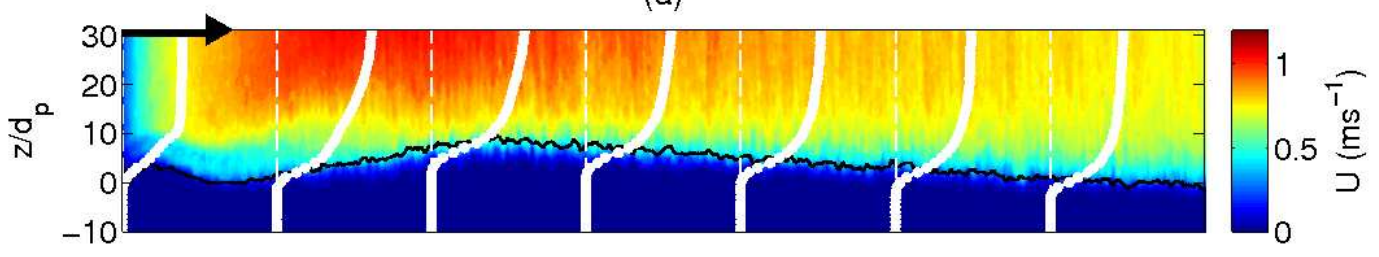

(b)

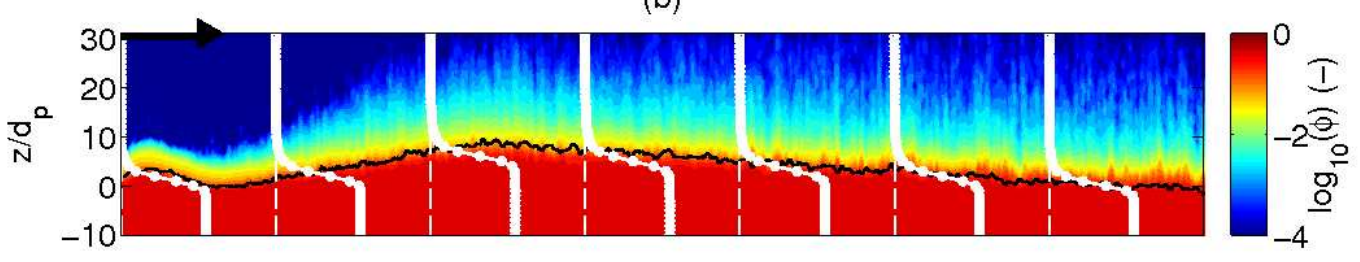

(c)

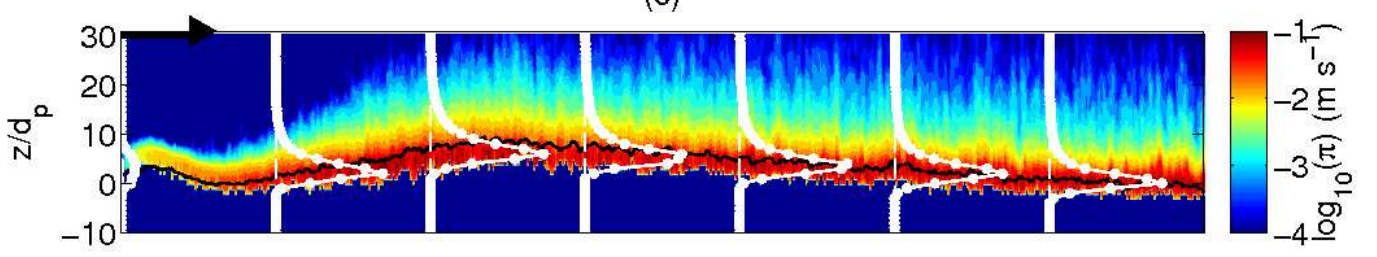

(d)

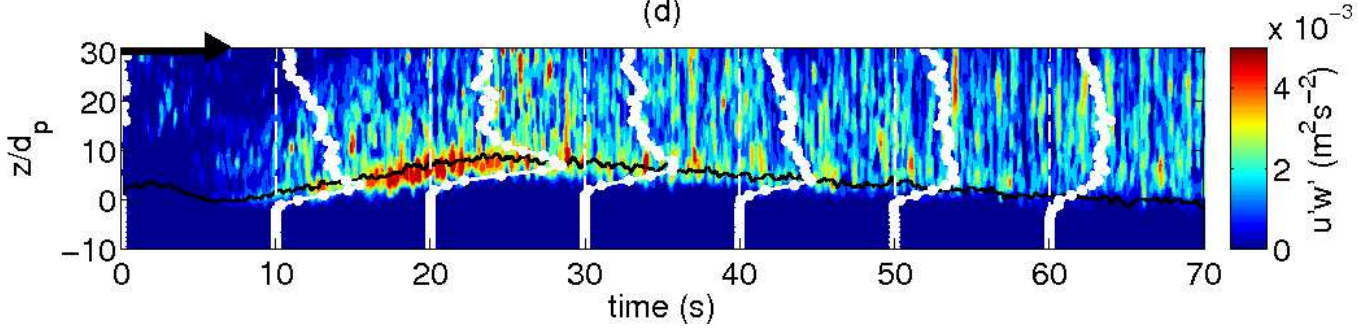

Figure 5. Colorplot of the ensemble averaged and filtered instantaneous streamwise velocity (a), concentration (b), 2D sediment flux (c) and absolute Reynolds stress (d). The ensemble averaged and filtered detected bed-interface positions is presented by the solid black lines. The vertical axis is made dimensionless by the particle diameter. The curves show the ensemble averaged vertical profiles of (a) streamwise velocity, (b) concentration, (c) sediment flux and (d) absolute Reynolds stress averaged over $6 \mathrm{~s}$ and represented every $10 \mathrm{~s}$. For these profiles the arrow at the top left corner of each panel corresponds to $u=1 \mathrm{~ms}^{-1}, \phi=0.55, \pi=0.05 \mathrm{~ms}^{-1}$ and $\left\langle u^{\prime} w^{\prime}\right\rangle=0.003 \mathrm{~m}^{2} \mathrm{~s}^{-2}$ respectively.

representative of the largest turbulent flow scales since $\Delta t \approx 30 T_{L S S}$ where $T_{L S S} \approx$ $0.7 H_{f} / U$ represents a characteristic scale of the time spent by the biggest turbulent structure in the measurement volume (Muste et al. 2004).

The selection of the adequate $6 \mathrm{~s}$ time interval in the quasi-steady bed erosion phase of the individual runs (i.e. for $t>30 \mathrm{~s}$ ) is based on the appreciation of the ensemble averaged data shown in figures 5 . In addition to the steady flow conditions, the selected $6 \mathrm{~s}$ time interval must also satisfy uniform flow conditions in contrast to accelerated or decelerated flow regimes. The velocity, concentration and sediment flux time series in figure 5 show that the most steady time interval is seen between $t=40 \mathrm{~s}$ and $t=50 \mathrm{~s}$. In this time interval, both the magnitudes of the measured quantities at a given distance from the bed and the shape of the vertical profiles are very similar. The mean absolute 
Reynolds shear stress is shown in figure $5 \mathrm{~d}$. In terms of flow uniformity, it can clearly be observed that the vertical profiles exhibit the most linear trend versus $z$ at the time $t=40 \mathrm{~s}$. Before and after this time, the vertical profiles follow respectively, a concave and a convex shape. Song \& Graf (1994) and Yang \& Chow (2008) showed that the deviation from a linear shear stress profile is an accurate indication of the flow non-uniformity. This is further confirmed here since the concave shear stress profile is associated with an accelerated bulk flow whereas the convex shape is obtained during the decelerating phase for $t \gtrsim 50 \mathrm{~s}$. As a consequence, the $6 \mathrm{~s}$ time interval over which the flow steadiness will be tested quantitatively is taken as $[40-46 \mathrm{~s}]$ in figure 5 . The analysis of the statistical properties of the ensemble averaged velocity, concentration and streamwise sediment flux profiles $\langle u\rangle(z),\langle w\rangle(z), \phi(z)$ and $\pi(z)$ relies on the calculation of the statistical moments between $t_{1}=40 \mathrm{~s}$ and $t_{2}=46 \mathrm{~s}$ as :

$$
\begin{gathered}
<u>(z)=\frac{1}{N} \sum_{i=1}^{N}\left(\frac{1}{\Delta t} \int_{t_{1}}^{t_{2}} u_{i}(t, z) d t\right) \\
<w>(z)=\frac{1}{N} \sum_{i=1}^{N}\left(\frac{1}{\Delta t} \int_{t_{1}}^{t_{2}} w_{i}(t, z) d t\right) \\
\phi(z)=\frac{1}{N} \sum_{i=1}^{N}\left(\frac{1}{\Delta t} \int_{t_{1}}^{t_{2}} \phi_{i}(t, z) d t\right) \\
\pi(z)=\frac{1}{N} \sum_{i=1}^{N}\left(\frac{1}{\Delta t} \int_{t_{1}}^{t_{2}} u_{i}(z, t) \phi_{i}(z, t) \mathrm{d} t\right)
\end{gathered}
$$

with $i$ standing for the realisation number. Under the assumption of ergodicity, the equivalent period of time averaging for a steady flow is $N \times \Delta t=66 \mathrm{~s}$. Over this averaging interval, the first order moments have a bias error in the range of $1 \%$. The Reynolds components of the velocity are estimated as:

$$
\begin{gathered}
u_{i}^{\prime}(t, z)=u_{i}(t, z)-<u>(z) \\
w_{i}^{\prime}(t, z)=w_{i}(t, z)-<w>(z)
\end{gathered}
$$

The mean Reynolds shear stress $\rho_{f}\left\langle u^{\prime} w^{\prime}\right\rangle(z)$ is calculated as:

$$
\rho_{f}<u^{\prime} w^{\prime}>(z)=\rho_{f} \frac{1}{N} \sum_{i=1}^{N}\left(\frac{1}{\Delta t} \int_{t_{1}}^{t_{2}} u_{i}^{\prime}(t, z) w_{i}^{\prime}(t, z) d t\right)
$$

In order to check the flow steadiness over the time interval [40 - 46 s], the ensemble averaged streamwise velocity profile given by equation (2.2) is calculated over the time interval $[40-43 \mathrm{~s}$ ] and compared to the one obtained over the time interval [ $43-46 \mathrm{~s}]$. As shown in the left panel of figure 6, the similarity of the two profiles further confirms the steadiness of the flow over the time interval [40-46 s]. A mixing layer induced by the vacuum box is observed on this plot $\left(z / d_{p}>40\right)$, but it represents only $20 \%$ of the total water depth. In the middle panel of figure 6 the profile of streamwise velocity averaged over the time interval [ $40-46 \mathrm{~s}$ ] for each of the 11 realisations is shown. The very good agreement between the different profiles in terms of magnitude and shape along $z$ further supports the high degree of experiment repeatability. Finally, the quantitative validation of the flow uniformity condition is based on the representation in figure 6 (right panel) 
(a)

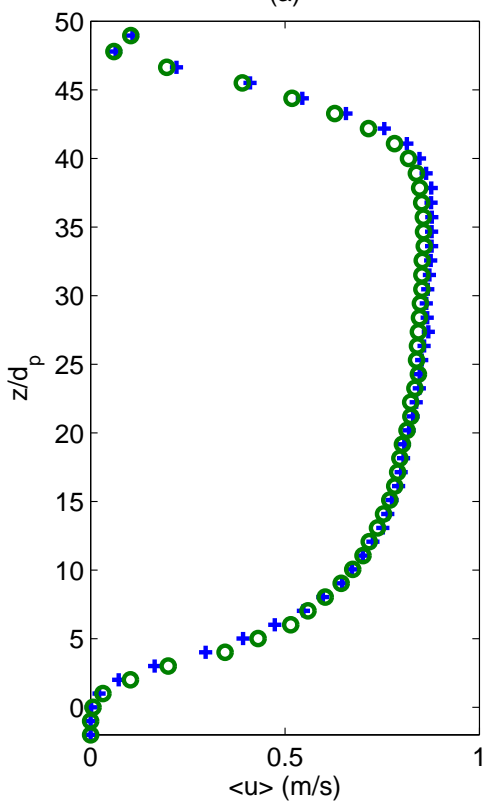

(b)

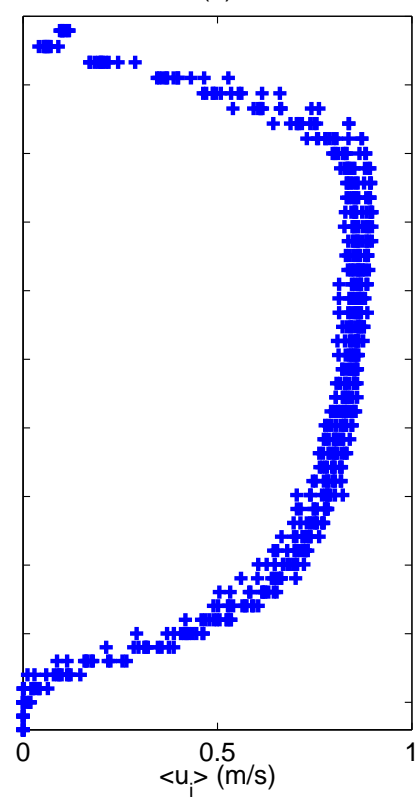

(c)

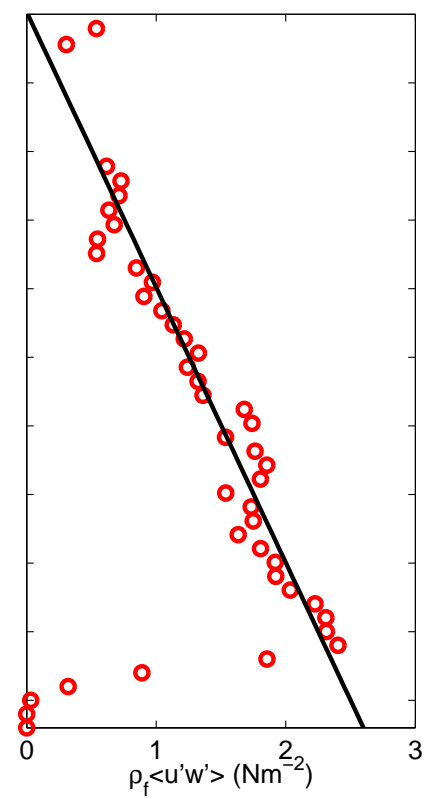

Figure 6. (a) Mean streamwise velocity profiles averaged over $t \in[40-43] \mathrm{s}(+)$ and over $t \in[43-46] \mathrm{s}(\mathrm{o})$. (b) Mean streamwise velocity profiles averaged over $t \in[40-46] \mathrm{s}$ for the 11 realisations. (c) Absolute Reynolds shear stress $\rho_{f}<u^{\prime} w^{\prime}>(\mathrm{o})$ and linear fit (-).

of the ensemble averaged absolute Reynolds shear stress profile given by equation (2.8). As previously observed in figure 5, this profile exhibits a linear evolution versus $z$ over the bulk flow region which is a clear indication of an established uniform flow (Yang \& Chow 2008). The linear fit of the shear stress profile (solid black line in figure 6c) crosses the zero axis at the vertical coordinate $z / d_{p}=50$. This position corresponds to a physical distance of $17 \mathrm{~cm}$ above the bed interface which is in very good agreement with the value of the measured flow depth. This aspect further indicates the full development of the shear boundary layer over the entire flow depth. In this section, the high degree of repeatability, steadiness and uniformity of the present experiment over the selected averaging interval of $[40-46 \mathrm{~s}]$ has been demonstrated. These data are used in the following to investigate the vertical structure of the uniform steady sheet flow.

Concerning the acoustic measurement uncertainties, Hurther \& Lemmin $(2001,2008)$ showed that the relative uncertainty in the mean velocity provided by a pulse-coherent ADVP technology (similar to the ACVP technology applied herein) is of the order of $1 \%$ due to the misalignement errors of the sensors in the submillimetre range. The nearbed velocity measurement is dependent on the bed interface detection, therefore the measurement uncertainty close to the bed can not be trivially evaluated. Nevertheless, the obtained accuracy of the measured transported volume for different flow conditions (see table 2) suggests negligibly low systematic errors in the velocity and concentration measurements. Considering the statistical bias error of $15 \%$ for the instantaneous concentration measurement, the relative uncertainty on the mean concentration data reduces to 1\% (Thorne \& Hurther 2014). The use of a central derivative scheme leads to relative uncertainties on the velocity gradients below $16 \%$ for $z / d_{p}<10$ and which reach $40 \%$ for $z / d_{p}=14$. Above this position the relative uncertainty diverges to infinity since the ve- 
(a)

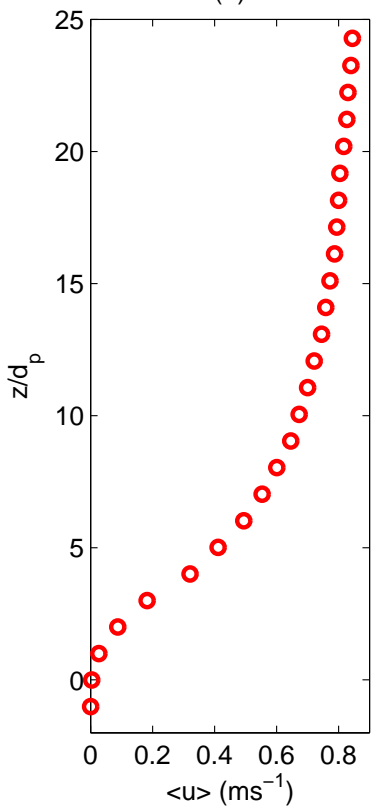

(b)

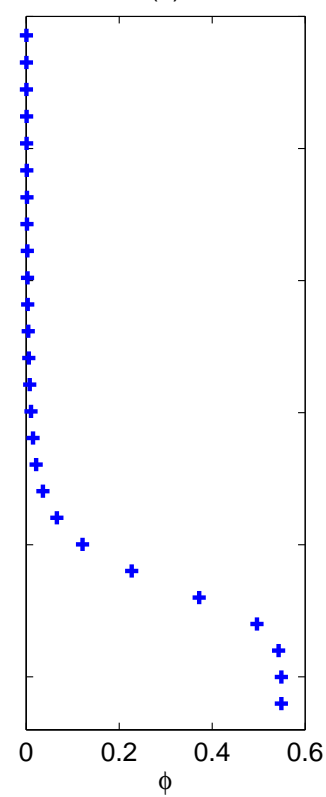

(c)

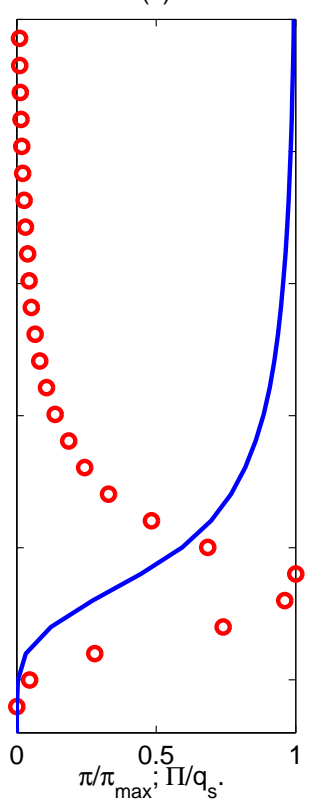

(d)

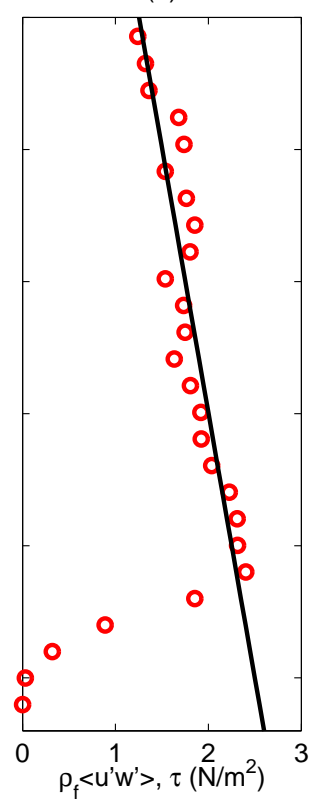

Figure 7. (a) Mean streamwise velocity profile. (b) Mean concentration profile. (c) Sediment flux (o) and cumulative sediment flux (-) per unit width, normalised by their maximum value. (d) Absolute Reynolds shear stress (o) and total shear stress (-).

locity gradients vanished. Concerning the concentration gradient, the relative uncertainty is below $5 \%$ for $z / d_{p}<14$.

\section{Results}

In this section, we present and discuss the vertical profiles of velocity, concentration, sediment flux and turbulent shear stress measured with the methodology described in the previous section. Moreover, the profiles of momentum and concentration diffusivities are presented and the vertical structure of the flow is analysed in terms of mixing length, Stokes and inertial numbers. Finally, the measurements are compared to different modelling approaches found in the literature.

\subsection{Mean profiles}

Figure 7 shows the vertical profiles of streamwise velocity (a), volumetric concentration (b), sediment flux repartition (c) and absolute Reynolds shear stress (d). For all figures, the vertical axis $z$ is made dimensionless by the particle diameter and its origin is taken at the vertical position where the mean streamwise velocity is lower than $1 \%$ of the bulk flow velocity $U$. This position is denoted as the bed interface in the following.

Just above the bed interface the velocity profile shown in figure 7a increases exponentially with the vertical distance to the bed. Above this exponential layer the velocity profile exhibits a linear behaviour including an inflection point. In the region $5 \lesssim z / d_{p} \lesssim 14$, the vertical profile follows a logarithmic shape.

The concentration profile presented in figure $7 \mathrm{~b}$ shows that concentration decreases linearly for $2 \lesssim z / d_{p} \lesssim 4-5$ whereas for $z / d_{p} \gtrsim 4-5$ the concentration decreases exponentially with $z$. This behaviour will be discussed in the following subsection. 
In order to study the solid load repartition along the vertical direction, the cumulative profile of volumetric sediment flux is evaluated over the interval [40-46 s] as

$$
\Pi(z)=\int_{-\infty}^{z} \pi(z) d z .
$$

Figure 7c presents both the vertical distribution of the sediment flux $\pi(z)$ and the cumulative flux $\Pi(z)$, normalised by their maximum values. A peak of sediment flux is seen at the position $z / d_{p} \approx 4$. The representation of the cumulative sediment flux shows that roughly half of the total solid load is transported below the position $z / d_{p} \approx 4-5$, where the concentration profile is linear and the other half is transported in the above lying layer over which the concentration profile decreases exponentially.

As mentioned previously, the linearity of the turbulent Reynolds stress with $z$ indicates the high degree of flow uniformity (figure 7d). Under uniform flow conditions, the streamwise momentum budget for the fluid-particle mixture reads (Revil-Baudard \& Chauchat 2013):

$$
0=\frac{\mathrm{d} R_{x z}^{f}}{\mathrm{~d} z}+\frac{\mathrm{d} \tau_{x z}^{p}}{\mathrm{~d} z}+\rho_{m} g S_{f},
$$

where $S_{f}$ is the friction slope, $\tau_{x z}^{p}$ is the intergranular shear stress, $R_{x z}^{f}$ is the turbulent Reynolds shear stress and $\rho_{m}=(1-\phi) \rho_{f}+\phi \rho_{p}$ is the mixture density. The viscous contribution can be neglected as shown by Revil-Baudard \& Chauchat (2013). By integrating equation 3.2 between a given position $z$ and the free surface $z=H_{f}$ the momentum balance can be written as:

$$
R_{x z}^{f}(z)+\tau_{x z}^{p}(z)=\tau(z)=\rho_{m} g S_{f}\left(H_{f}-z\right) .
$$

Assuming that the granular contribution is negligible in the upper part of the flow $\left(z / d_{p} \gtrsim\right.$ 5 ), the single contribution to the total shear stress is the turbulent one. Therefore, the linear fit of the measured turbulent shear stress in the upper part of the flow can be used to evaluate the total shear stress profile $\tau(z)$ over the entire water column, from the free surface down to the bed interface. The value of the total shear stress at the bed interface provides the friction velocity as: $\tau(z=0)=\rho_{f} u_{*}^{2}$. This method gives a value of $u_{*}=5.0 \mathrm{~cm} / \mathrm{s}$ corresponding to a Shields number value of $\theta=0.44$. This indicates a sediment transport regime at the transition between the bed-load and the sheet flow regime. The corresponding suspension number value is $S=w_{s} / u_{*}=1.1$ indicating a transport regime at the transition between the suspension and the no-suspension mode (Sumer et al. 1996).

Another classical method to evaluate the friction velocity is the momentum balance for the water column. This leads to the well-known energy slope formulation as $u_{*}^{m}=$ $\left(g r_{b} S_{0}\right)^{1 / 2}=6.2 \mathrm{~cm} / \mathrm{s}$, where the equivalent hydraulic radius $r_{b}$ is evaluated with the method proposed by Vanoni \& Brooks (1957) for the side wall corrections. With this estimate of the friction velocity, the Shields number becomes $\theta^{m}=0.68$. It must be reminded that the classical log-fit method for the evaluation of the friction velocity is not adapted to the sheet-flow conditions investigated herein. The application of this method to mobile bed flow conditions is ambiguous since the origin of the vertical axis is chosen arbitrary and the von Karman constant is subject to variations around the value of 0.41 due to the presence of moving particles (Vanoni 1975). This point will be discussed in details in the following. Regarding the difference of about $20 \%$ between the two different friction velocity estimation, Hurther et al. (2007) obtained similar differences under uniform and fixed rough bed flow conditions. Moreover, van der A et al. (2011) demonstrated the validity of the Reynolds bed shear stress method by comparing it to 
the classical log-fit method. Very good agreement were found in their turbulent rough bed oscillatory flow for the low roughness case (fixed sand particles).

The total solid load per unit width is computed from equation 3.1 as $q_{s}=\Pi(H f)=$ $7.1 \times 10^{-3} \mathrm{~m}^{2} / \mathrm{s}$ and in dimensionless form as:

$$
\psi=q_{s} / \sqrt{\left(\rho_{p}-\rho_{f}\right) g d_{p}^{3} / \rho_{f}}=3.2 .
$$

This value can be compared with the result of Meyer-Peter \& Muller (1948)'s formula $\psi=8\left(\theta-\theta_{c}\right)^{3 / 2}$, where $\theta_{c}=0.05$ is the critical threshold value for the initiation of particle motion. Using $\theta=0.44$, the resulting dimensionless solid load is $\psi=1.9$ which is $40 \%$ lower than the measured value. This value lies within the range of variation seen in Meyer-Peter \& Muller (1948)'s measurements. These experimental data were subject to large measurements uncertainties for the solid load and the bed shear stress estimates and therefore we consider this comparison as good. Moreover, if the larger Shields number of $\theta^{m}=0.68$ is used, the dimensionless solid load becomes $\psi=5$ which again lies within the range of uncertainties seen in the data of Meyer-Peter \& Muller (1948). This further confirms the validity of the coupled sediment transport and flow forcing measurements provided by the ACVP technology.

\subsection{Vertical structure of the flow}

When sediment particles are transported in a turbulent boundary layer, streamwise momentum is diffused downward from the bulk flow toward the fixed sediment bed while sediment concentration is diffused upward from the dense moving bed toward the dilute suspension. The nature of these mixing processes depends on the local properties of the flow and a quantitative understanding of these mechanisms is the key issue for an accurate modelling of sediment transport.

Assuming that the shear stress follows a Fickian law, i.e the momentum flux is proportional to its spatial derivative, one can write the following relationship for the total shear stress:

$$
\tau=\rho_{m} \epsilon_{m}\left|\frac{\mathrm{d} u}{\mathrm{~d} z}\right|,
$$

where $\epsilon_{m}$ represents the momentum diffusivity which has the dimension of a kinematic viscosity. As explained above, the vertical profiles of total shear stress $\tau(z)$ is obtained by a linear fit of the turbulent Reynolds shear stress measurements in the dilute region (see figure 7). The vertical profile of mean velocity shear rate $\frac{\mathrm{d} u}{\mathrm{~d} z}$ is calculated from the mean velocity profile. Consequently, the vertical profile of momentum diffusivity $\epsilon_{m}(z)$ can be evaluated as:

$$
\epsilon_{m}=\frac{\tau}{\rho_{m}\left|\frac{\mathrm{d} u}{\mathrm{~d} z}\right|},
$$

where the spatial derivative in $z$ is approximated by a central derivative scheme.

Following the idea originally introduced by Rouse (1937), the sediment volume balance in the vertical direction can be written as an equilibrium between the downward settling flux $w_{s} \phi$ and an upward turbulent dispersion or resuspension flux $\epsilon_{p} \frac{d \phi}{d z}$. Using a similar Fickian approach for the particle flux, the following equation is obtained:

$$
w_{s} \phi-\epsilon_{p} \frac{\mathrm{d} \phi}{\mathrm{d} z}=0 .
$$

The concentration diffusivity $\epsilon_{p}$ can be evaluated from the mean concentration profile as: 


$$
\epsilon_{p}=\frac{w_{s} \phi}{\frac{\mathrm{d} \phi}{\mathrm{d} z}},
$$

where the vertical gradient of concentration is also computed using a finite difference central scheme.

Following the concept of mixing length in the wall region (Prandtl 1926), where the law of the wall relates the eddy viscosity to the most energetic turbulent eddy size represented by the mixing length $l_{m}$, the momentum diffusivity $\left(\epsilon_{m}\right)$ defined in equation 3.6 can be expressed as a function of this characteristic eddy length scale as:

$$
\epsilon_{m}=l_{m}^{2}\left|\frac{\mathrm{d} u}{\mathrm{~d} z}\right| .
$$

Substituting equation 3.6 in 3.9 one can compute the mixing length profile as:

$$
l_{m}=\frac{\sqrt{\tau / \rho_{m}}}{\left|\frac{\mathrm{d} u}{\mathrm{~d} z}\right|} .
$$

It must be noticed that the momentum diffusivity has a more generic meaning than the eddy viscosity provoked by turbulent flow eddies. In fact, it can be anticipated that the momentum diffusivity in the dilute region of the flow will actually correspond to the eddy viscosity where $l_{m}$ should follow a linear evolution with $z$. However, in the dense region of the flow the momentum diffusivity will be dominated by intergranular interactions. According to Bagnold (1954) the shear stress associated with intergranular interactions in the grain inertia regime is proportional to $\rho_{p} d_{p}^{2}|\mathrm{~d} u / \mathrm{d} z|^{2}$ corresponding to a length scale of momentum diffusion controlled by the particle diameter $d_{p}$. A particulate viscosity has also been introduced by Chauchat \& Médale (2010) and Chauchat \& Médale (2014) for numerical simulations of dry or immersed granular flows based on the dense granular flow rheology $\mu(I)$. It is noteworthy that the particulate viscosity is expected to diverge in the granular flow region in quasi-static regime.

Figure 8 presents the normalised velocity and concentration profiles (a), the vertical evolution of momentum and concentration diffusivities (b) and the vertical profile of mixing length (c). It can be seen that the momentum diffusivity diverges to infinity close to the bed interface, characterising the transition from a fluid-like to a solid-like behaviour. As expected it reaches a minimum corresponding to the inflection point of the velocity profile. For $z / d_{p} \gtrsim 5$, the momentum diffusivity is equivalent to an eddy viscosity and increases linearly with $z$. The concentration diffusivity follows the same trend but is always higher than the momentum diffusivity.

As can be seen in figure $8 c$, the mixing length diverges downward for $z / d_{p}<2$, consistently with the momentum diffusivity profile. This indicates that the characteristic length scale associated with momentum diffusion increases dramatically downward. As proposed by Jenkins (2007), this behaviour is most probably related to the increase of the length of contact chains when enduring contacts occur between particles. The length scale associated with the contact chain network seems to be the relevant length scale of momentum diffusion in this layer. This suggests that momentum is mainly transferred through permanent or long term contacts between particles by frictional interactions. In the region $2 \lesssim z / d_{p} \lesssim 4-5$, the mixing length reaches a minimum $\left(l_{m} / d_{p} \approx 0.5\right)$ and is fairly constant. The mixing length is a fraction of the particle diameter in this region which suggests that the granular flow corresponds to the grain inertia regime. This is consistent with the granular shear stress formulation proposed by Bagnold (1954). Intu- 
(a)

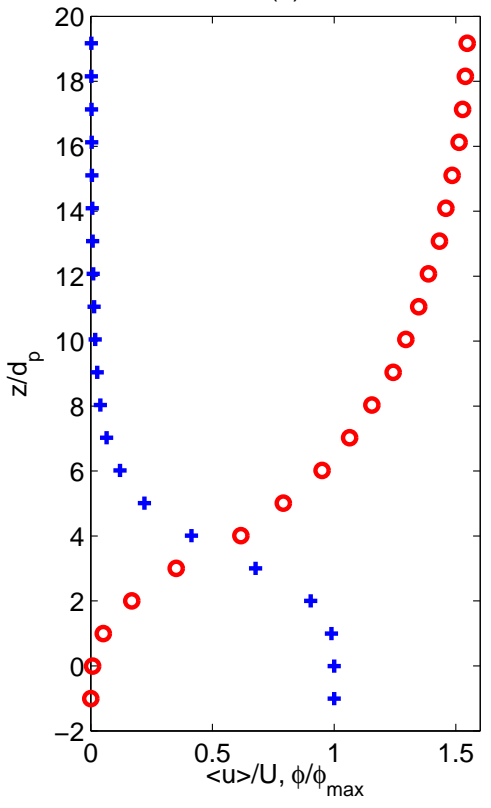

(b)

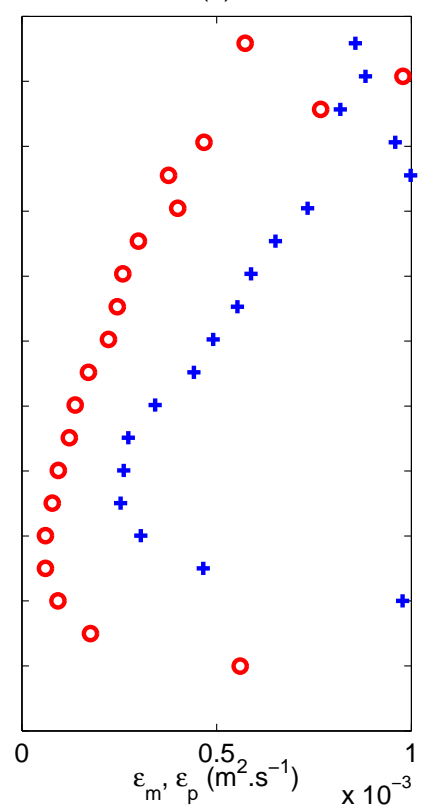

(c)

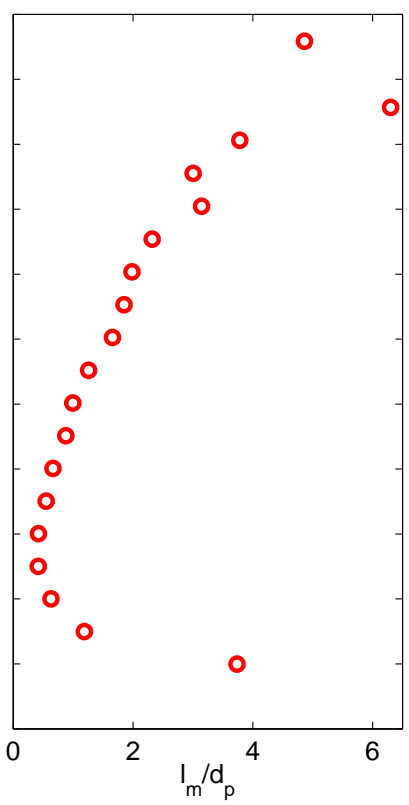

Figure 8. (a) Normalised mean streamwise velocity (o) and concentration profiles (+). (b) Momentum (o) and concentration ( + ) diffusivities. (c) Mixing length profile.

itively, the close agreement between the particle motion scale and their diameter suggests that they interact through collisions. For $z / d_{p} \gtrsim 4-5$, the mixing length profile increases linearly with $z$, consistently with the law of the wall in turbulent shear boundary layers. This observation supports the fact that the large-scale turbulent motions are the dominant momentum diffusion scales in this region.

From this analysis, the position $z / d_{p} \approx 4-5$ corresponds to the transition between a layer dominated by granular interactions, denoted as the bed layer in the following, and a layer dominated by turbulent processes, denoted as the suspension layer in the following.

In order to further precise the nature of intergranular interactions in the bed layer, the Stokes and Inertial numbers, characterising the granular flow regime, are introduced. Following Armanini et al. (2005)'s definition a Stokes number based on the velocity shear rate is defined as:

$$
S t=\frac{1}{18} \frac{\rho_{p}}{\rho_{f}} \frac{d_{p}^{2}\left|\frac{\mathrm{d} u}{\mathrm{~d} z}\right|}{\nu_{f}} .
$$

This Stokes number characterises the competition between the deformation rate at the origin of particle fluctuating motions and the viscous effects that damp collisions efficiency. For $S t \gtrsim 10-15$, the fluctuating motion of particles is no longer influenced by the fluid viscosity and inertial collisions is the dominant mechanism. On the other hand for $S t \lesssim 10-15$, viscous effects are strong enough to damp collisions efficiency and reduce dramatically particle velocity fluctuations (Armanini et al. 2005). Figure 9b shows the profile of the Stokes number. The profile shape and the order of magnitude is in good agreement with those obtained by Armanini et al. (2005) for a different particle 
(a)

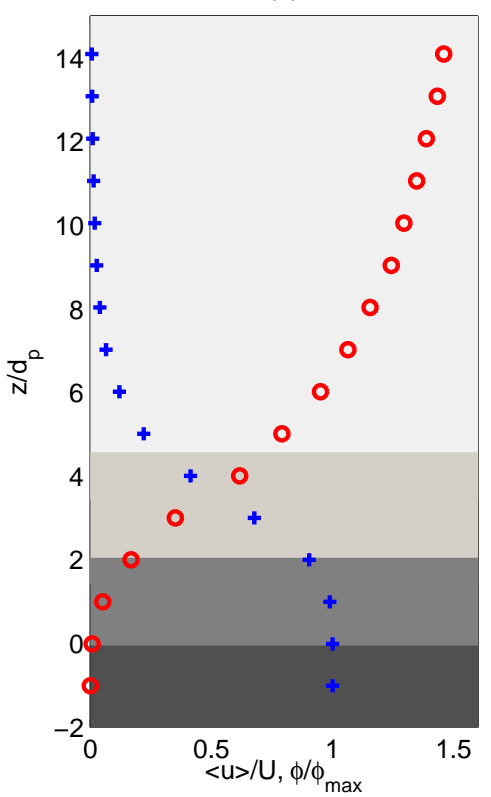

(b)

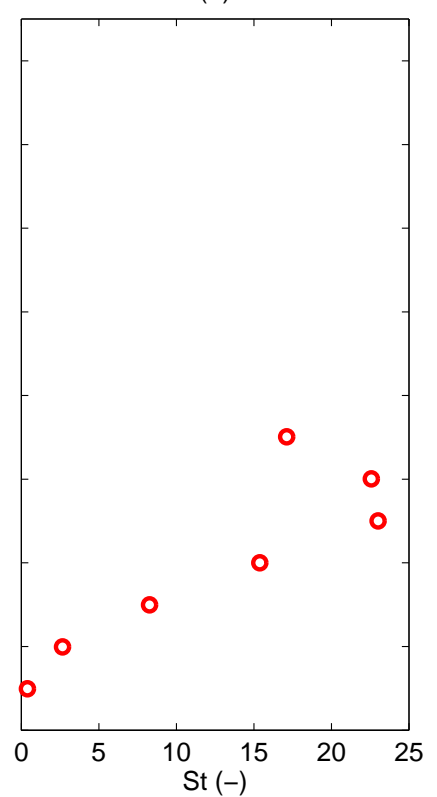

(c)

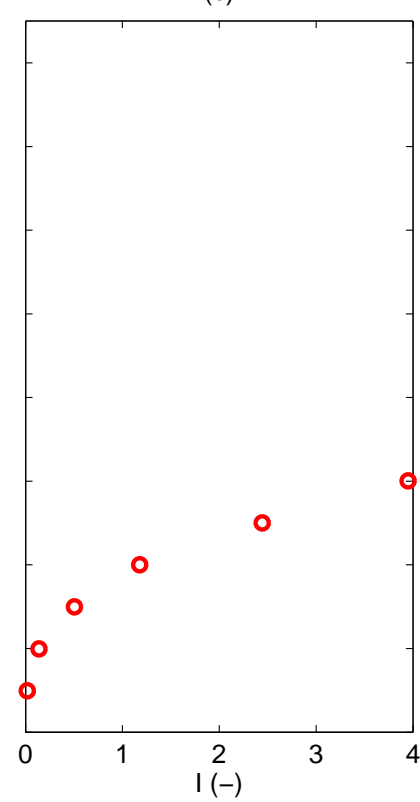

Figure 9. (a) Normalised mean streamwise velocity (o) and concentration profiles (+) and representation of the static, frictional, collisional and suspension layers (from dark to light). (b) Stokes number profile. (c) Inertial number profile.

shape and density. In the present experiment the Stokes number is greater than 10-15 for $z / d_{p} \gtrsim 2$. This confirms that the granular flow in this layer is collisional.

In addition to this first Stokes number a second one is defined as the ratio between a free-fall time scale and a viscous time scale for a particle in a dense granular media submitted to a particulate pressure $P^{p}$ in a fluid of viscosity $\nu_{f}$ (Cassar et al. 2005):

$$
S t^{*}=\frac{d_{p}}{\nu_{f}} \sqrt{\rho_{p} P^{p}}
$$

This Stokes number has been introduced in the framework of the dense granular flow rheology and it allows to determine whether the timescale of rearrangement is controlled by the fluid viscosity or the particle inertia. The corresponding regimes are the viscous or inertial regimes of the granular flow respectively (Andreotti et al. 2013). The particulate pressure $P^{p}$ represents the normal stress associated with the granular interactions. Under steady uniform and homogeneous conditions and for concentration values higher than a given critical concentration $\phi_{c}$, the particulate pressure balances the buoyant weight of particles above a given position $z$ such that:

$$
P^{p}(z)= \begin{cases}0 & \text { for } \mathrm{z} \geqslant \mathrm{z}_{\mathrm{c}} \\ \left(\rho_{p}-\rho_{f}\right) g \int_{z}^{z_{c}} \phi(z) \mathrm{d} z & \text { otherwise }\end{cases}
$$

where $z_{c}$ is the vertical position at which $\phi=\phi_{c}$. In the present case we have set the critical concentration $\phi_{c}=0.08$ corresponding to an inter-particle distance of one particle diameter. This value is commonly used as the transition below which intergranular stresses can not be neglected (e.g. Hsu et al. 2004). This criteria corresponds here to the position $z_{c} / d_{p} \approx 5$ which is in good agreement with the transition found on the basis of 
momentum diffusivity analysis (figure 8). This further confirms that this position represents a transition between the bed and suspension layers. One must notice that equation 3.13 is valid only if granular interactions are the only processes at work inside the bed layer and that buoyant weight of particles is entirely supported by intergranular normal stress i.e. turbulent effects do not participate to particle dispersion for $z / d_{p}<5$. Based on this estimate of the particulate pressure the value of $S t^{*}$ is always larger than $10^{2}$ in the bed layer, indicating that the granular flow is in the inertial regime and that the dense granular flow rheology is governed by the inertial number $I$ defined as:

$$
I=\frac{d_{p}\left|\frac{\mathrm{d} u}{\mathrm{~d} z}\right|}{\sqrt{P^{p} / \rho_{p}}} .
$$

The inertial number can be interpreted as the ratio between the vertical time scale of rearrangement and the horizontal time scale of deformation (Andreotti et al. 2013). For $I \approx 0$ the granular flow is in the quasi static regime. For $I \gtrsim 1$, the granular flow is in the gaseous regime in which binary collisions are dominant (Forterre \& Pouliquen 2008). In between these two values the granular flow is in the liquid regime where both frictional and collisional interactions control the flow behaviour.

In figure $9 \mathrm{c}$ the vertical profile of the inertial number is presented. One can observe that $I$ increases from zero to one between $z / d_{p}=0$ and $z / d_{p} \approx 2$. For $z / d_{p}>2$, I still increases and reaches values greater than unity. This is in agreement with the conclusions raised by Armanini et al. (2005) and Capart \& Fraccarollo (2011) that in the lower part of the bed layer frictional interactions are dominant whereas in the upper layer binary collisions are dominant. The thickness of the frictional layer of about $2 d_{p}$ observed in the present experiment is in good agreement with the measurements of Capart \& Fraccarollo (2011) for the same Shields number.

To summarise the main findings presented in this subsection, it has been shown that a transition from a suspension layer dominated by turbulent mechanisms to a bed layer dominated by granular interactions occurs around $z / d_{p} \approx 4-5$. The bed layer can be divided into two sublayers, an upper layer in which binary collisions dominate the vertical transfer of momentum and a lower layer in which frictional interactions dominate. Furthermore, for a suspension number around unity the solid load is equipartitioned between the bed layer and the suspension layer.

\subsection{Turbulent processes in the suspension layer}

In this subsection, the modulation of turbulent momentum-mixing efficiency due to the presence of particles is first considered by comparing the direct mixing length estimation to different formulations taking into account sediment effects. Second, the concentration profile obtained from a Rouse model is compared with the one measured in the suspension layer.

\section{Mixing length profile}

Figure 10a shows mixing length profile deduced from the experimental measurements. As mentioned previously the mixing length is linear in $z$ for $z / d_{p} \in[5 ; 14]$ and can be approximated by the following expression as:

$$
l_{m}(z)=\kappa^{S F}\left(z-z_{d}\right)
$$

in which the Von Karman parameter $\kappa^{S F}=0.225$ and the mixing length origin $z_{d}=$ $3.1 d_{p}$ have been determined from a best fit. The solution is represented by a blue solid 
(a)

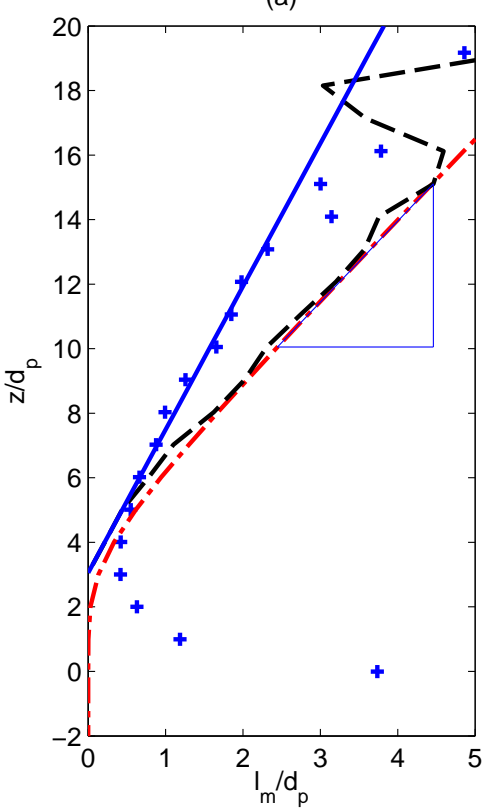

(b)

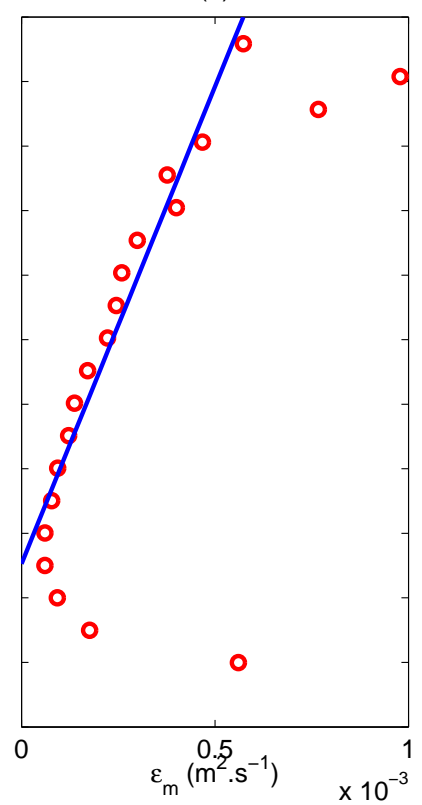

(c)

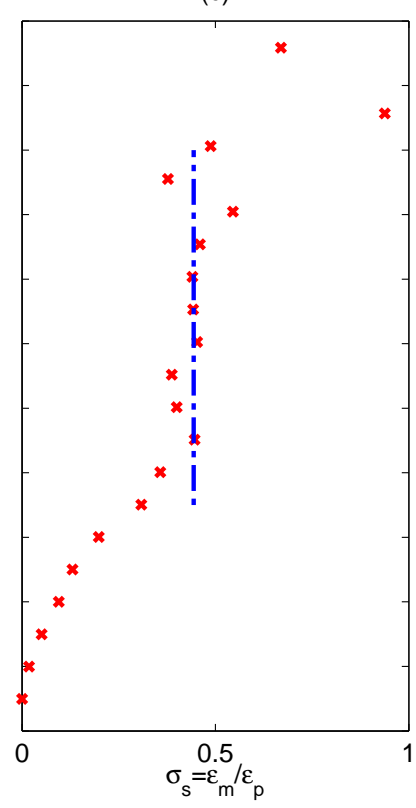

Figure 10. (a) Mixing length profiles: measured $(+)$, linear fit $(-)$, result of equation 3.16 (- - -) and result of equation 3.18 (- . -). The coefficient of determination of the linear fit is $R^{2}=0.984$. The slope 0.41 is represented by the blue triangle. (b) Profile of measured momentum diffusivity (o) and linear fit obtained from the mixing length linear fit (-). (c) Ratio between the momentum and concentration diffusivity $(\mathrm{x})$. The vertical dot-dashed line corresponds to the Schmidt number value and the associated standard deviation is 0.05.

line in figure 10a. The obtained value of $\kappa^{S F}$ is significantly lower than the value obtained in clear water turbulent boundary layers $\left(\kappa^{c w}=0.41\right)$. This feature is representative of a strong turbulence damping induced by the presence of particles as previously observed by Vanoni (1975); Best et al. (1997); Amoudry et al. (2008) and Gaudio et al. (2010) amongst others. It is noteworthy that the mixing length slope remains as low in the upper water column where the local concentration values are particularly low. This suggests that the effect of particles in the lower part affects the mixing length associated with turbulent eddies over a large fraction of the boundary layer, even in regions where the concentration is almost zero. This supports the presence of non local effects induced by the sheet flow layer.

From a modelling point of view, the turbulence damping induced by the presence of particles in sediment laden flows has been classically considered via stratification effects (Villaret \& Trowbridge 1991). The same conceptual approach has been applied in several sheet flow models to account for turbulence damping effects (Jenkins \& Hanes 1998; Capart \& Fraccarollo 2011; Berzi \& Fraccarollo 2013). In this approach, a correction depending on the Richardson number is introduced as:

$$
l_{m}^{R i}=\sqrt{1-\phi}(1-7 R i) \kappa^{c w}\left(z-z_{d}\right)
$$

where a shifted vertical axis $z-z_{d}$ is used consistently with the best fit method applied previously. The Richardson number characterises the competition between turbulence production induced by shearing and turbulence damping induced by density stratification 
as:

$$
R i=\frac{-\frac{g}{\rho_{m}} \frac{d \rho_{m}}{d z}}{\left|\frac{\mathrm{d} u}{\mathrm{~d} z}\right|^{2}}
$$

The Richardson number decreases rather linearly from $R i=0.09$ at $z / d_{p}=0$ to $R i=0.01$ at $z / d_{p}=15$ (not shown here). The resulting mixing length profile (equation 3.16 ) is shown in figure 10a (black dashed line). It can be seen that this formulation underestimates the damping effect because the resulting mixing length profile is larger than the measured one. Furthermore, as the correction factor is restricted to local stratification effects, which are very low above $z / d_{p} \approx 5$, it cannot account for the non-local effects supported by the direct mixing length measurement. This is illustrated by the fact that the slope of the modelled mixing length reaches the clear water value 0.41 immediately above the bed layer. The Von Karman parameter used in formulation 3.16 has been taken at its clear water value as it is classically done in a priori modelling approaches.

The empirical formulation used by Revil-Baudard \& Chauchat (2013) in their twophase sheet flow model, relates the mixing length to the integral of the concentration profile according to:

$$
l_{m}^{\phi}=\kappa^{c w} \int_{-\infty}^{z} \frac{\phi^{m}-\phi}{\phi^{m}} d z .
$$

This formulation is a first order model approximation taking into account non-local effects. The corresponding profile is represented by the red solid line in figure 10a. An interesting aspect of this simple model is that unlike equation 3.16 no reference of the bed level position is required, as long as the concentration profile is known. It can be seen on figure $10 \mathrm{a}$ that the integral of the concentration profile slightly modifies the slope of the modelled mixing length up to the position $z / d_{p} \approx 7$. However, the modelled mixing length values are also overestimated compared to the direct estimation, suggesting that this first order approach is not sufficiently accurate without tuning the von Karman parameter.

It appears that the modification of the mixing length in regions where the concentration and the stratification are negligibly low implies that more refined turbulence models are required.

\section{Concentration profile}

The analytical expression of the concentration profile in the suspension layer can be obtained from the sediment mass balance (see equation 3.7, Rouse 1937). The upward turbulent dispersion flux is modelled by a concentration diffusivity $\epsilon_{p}$, related to the momentum diffusivity by the introduction of a Schmidt number $\sigma_{s}$ as : $\epsilon_{p}=\epsilon_{m} / \sigma_{s}$.

The Schmidt number corresponding to the ratio between the momentum diffusivity and the concentration diffusivity is shown in figure 10c. The ratio increases from zero at the bed interface to a fairly constant value of $\sigma_{s}=0.44$ for $z / d_{p} \gtrsim 5$. The region of constant Schmidt number value confirms the validity of the proportionality between the concentration diffusivity and the eddy viscosity assumed in the Rouse formulation. The systematic uncertainty on the Schmidt number is dominated by the dispersion in the settling velocity estimations (see equation 3.8). It is evaluated to $20 \%$, even though no size segregation has been observed in the suspension.

Using the linear fit of the mixing length profile (equation 3.15), the profile of momentum diffusivity in the suspension layer (see figure 10b) can be expressed as:

$$
\epsilon_{m}=u_{*} \kappa^{S F}\left(z-z_{d}\right)
$$




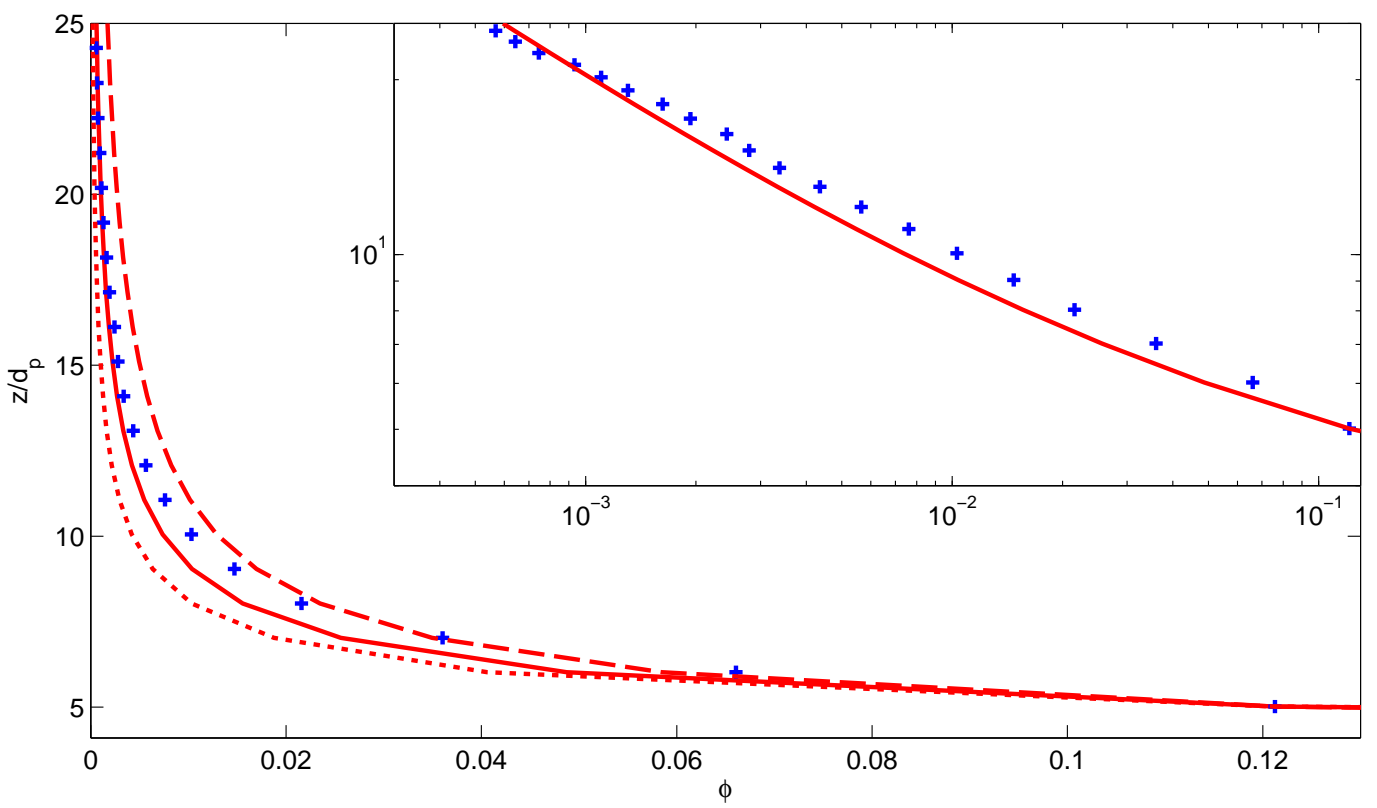

Figure 11. Comparison between measured $(+)$ and modelled suspension profiles using $\sigma_{s}=0.44$ (solid line), $\sigma_{s}=0.528$ (dotted line) and $\sigma_{s}=0.352$ (dashed line).

Substituting this relationship in equation (3.7) the sediment volume balance can be written as:

$$
\frac{\mathrm{d} \phi}{\mathrm{d} z}-\frac{p}{\left(z-z_{d}\right)} \phi=0
$$

where $p=-\frac{\sigma_{s} w_{s}}{\kappa^{S F} u_{*}}$ is the Rouse number as defined by Nielsen \& Teakle (2004). Equation (3.19) is a first order ordinary differential equation in $z$ that can be integrated analytically from a given elevation $z_{r}$ at which the concentration $\phi\left(z_{r}\right)=\phi_{r}$ is known. This leads to the well-known Rouse profile as:

$$
\phi(z)=\phi_{r}\left(\frac{z-z_{d}}{z_{r}-z_{d}}\right)^{p},
$$

Figure 11 shows the comparison between the concentration profile predicted by equation 3.20 using $\sigma_{s}=0.44 \pm 0.088, \kappa^{S F}=0.225, z_{d} / d_{p}=3.1, \phi_{r}=0.12$ and $z_{r} / d_{p}=5$ and the measurements of $\phi(z)$. The good agreement observed in this figure confirms the validity of an equilibrium between the settling flux and the turbulent dispersion flux in the suspension layer. However, one must keep in mind that i) a model is required to predict the von Karman parameter and the Schmidt number values, ii) a reference concentration is needed and iii) the profile strongly depends on the position of the vertical axis origin. The definition of this origin deserves a particular attention for a suspension layer in the presence of a thick sheet-layer.

\subsection{Granular processes in the bed layer}

In the following, the $\mu(I) / \phi(I)$ rheology (Forterre \& Pouliquen 2008) is compared with the measurements obtained in the bed layer. The local value of the Inertial number 
(a)

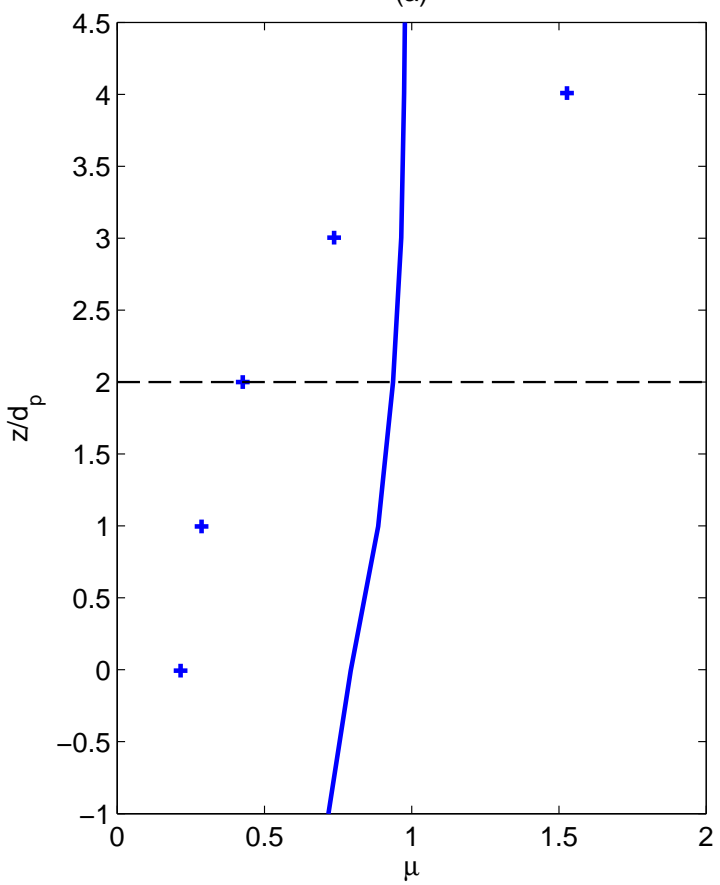

(b)

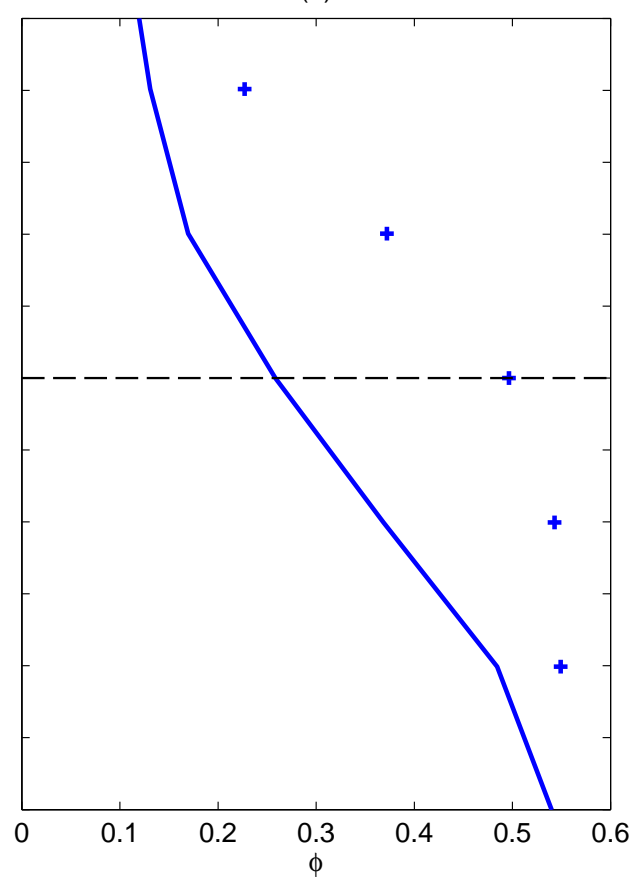

FiguRE 12. Comparison between measurements $(+)$ and prediction from the granular rheology $\mu(I) / \phi(I)$ (-) for the friction coefficient profile (a) and the concentration profile (b). The horizontal dashed line represents the position for which $I=1$.

(equation 3.14 and figure 9) is used to model the friction coefficient:

$$
\mu(I)=\mu_{s}+\frac{\Delta \mu}{I_{0} / I+1},
$$

where $\mu_{s}$ represents the static friction coefficient, the so-called tangent of the angle of repose, which has been determined experimentally herein $\left(\mu_{s}=0.7\right.$ see table 1$)$, $\Delta \mu$ is the difference between the static and dynamical friction coefficients and $I_{0}$ is a phenomenological parameter of the rheology. These parameters are set to $\Delta \mu=0.3$ and $I_{0}=0.3$, valid for for dry granular flows of glass beads in the inertial regime. These values are also consistent with the ones used in the two-phase sheet flow model proposed by Revil-Baudard \& Chauchat (2013).

Concerning the concentration profile, the $\phi(I)$ formulation is given by:

$$
\phi(I)=\frac{\phi^{m}}{1+I} .
$$

Figure 12a shows the comparison between the friction coefficient $\mu=\tau / P^{p}$ estimated from the measurements and the results from equation (3.21). The measurements indicate that $\mu$ increases with $z$ from a static value of $\mu \approx 0.2$ at $z / d_{p}=0$ to a value of $\mu \approx 1.5$ at $z / d_{p}=4$. The friction coefficient at the bed interface is much lower than the expected value of $\mu_{s}=0.7$. Such a difference has already been observed for laminar bed-load of spherical particles but in a smaller proportion (Aussillous et al. 2013). This discrepancy will be discussed in the next section. Moreover, the friction coefficient exceeds unity and keeps increasing until the top of the bed layer while the $\mu(I)$ law predicts a saturation at $\mu=\mu_{s}+\Delta \mu \approx 1$. A friction coefficient greater than one suggests that other mechanisms 
than contact interactions contributes to the shear stress. Boyer et al. (2011) have observed a similar behaviour when analysing their rheometer data of dense suspension. The authors suggested that the difference between the overall friction coefficient and the friction coefficient associated with contact interactions is due to hydrodynamic interactions linked to lubrication. In the present case, values of the friction coefficient greater than one is more likely to be associated with inter-particle collisions and/or turbulent velocity fluctuations. At this stage of the analysis, the origin of this point remains unclear.

Figure $12 \mathrm{~b}$ shows the comparison of the concentration profile predicted by equation (3.22) with the measurements. The agreement is rather poor and no concentration shoulder is observed contrary to the model prediction of Hsu et al. (2004) based on a kinetic theory approach or of Revil-Baudard \& Chauchat (2013) based on the granular flow rheology.

These discrepancies between the granular flow rheology and the measurements raise a number of questions: is the particulate pressure estimation based on the concentration profile valid? Are the assumptions of the granular rheology approach verified under sheet flow conditions? Concerning the particulate pressure, the estimate relies on the validity of the measured concentration profile in the denser part of the flow. Also the momentum balance between intergranular interactions and gravity inside the bed layer is based on the assumption that turbulent dispersion effects are negligible inside the bed layer. This is probably a too strong assumption. Finally, the actual value of the critical volume fraction $\phi_{c}$ is also subject to uncertainties. The choice of $\phi_{c}=0.08$ maximises the particulate pressure which can induce an underestimation of the friction coefficient. However, the factor of more than three observed for $\mu$ at the fixed bed interface can not be explained solely by this uncertainty.

\section{Discussion}

This section discusses the validity of the steadiness assumption on which the granular rheology is based and it further interprets the modification of the von Karman and Schmidt numbers.

\subsection{Intermittency of the bed layer}

An important assumption in the dense granular flow rheology is the steadiness of the granular flow at scales larger than the grain scale. The results presented above are based on average quantities consistently with this hypothesis. The experimental setup is used here to verify this assumption by making use of the high-rate profiling of velocity and concentration as provided by the ACVP technology. Figure 13 shows an example of the time evolution of the ACVP measurements over a duration of approximately 3 seconds acquired at a frequency of $78 \mathrm{~Hz}$. As mentioned in section 2, the bed interface detection and the concentration measurement are low-pass filtered at a frequency of $7.8 \mathrm{~Hz}$ and 4.9 $\mathrm{Hz}$ respectively to guaranty a low bias error. The blue and red solid contours represent isolines of relative turbulent shear stress identified as sweep and ejection events, respectively. The uw-quadrant threshold technique of Lu \& Willmarth (1973) has been applied here with a threshold value of $H=2$ as proposed by Mignot et al. (2009). The colorplot represents the base 10 logarithm of the concentration and the black solid line represents the detected bed interface position. The $2 \mathrm{C}$ vector plot corresponds to the instantaneous field of the $2 \mathrm{C}$ time fluctuating velocity. It confirms that the velocity field inside a red and blue delimited contour is oriented in quadrant 2 (ejection) and 4 (sweep) in the (u,w) plane, respectively. This figure illustrates the complex interactions between large-scale coherent flow structures, suspended sediment concentration and the bed interface dy- 
namics. A close examination of this figure reveals that erosion events, corresponding to a drop of the bed level position, are associated to sweep events. In terms of concentration, these erosive events are associated with a reduction of concentration while accretion events, corresponding to an increase of the bed level position, are linked to ejections events inducing an increase of concentration. Intuitively, it can be deduced that sweeps are mainly responsible for momentum diffusion while ejections are mainly responsible for particle dispersion/resuspension. This large scale turbulence driven intermittency is also believed to explain why the measured granular rheology deviates from the steady state rheology. Due to the intermittency under the action of the sweeps events, the dense frictional layer observed in the present experiment can be dominated by turbulent processes for the duration of this event. Indeed, on average the flow in the bed layer is highly concentrated and dominated by granular interactions, however turbulent processes occur on short duration and are sufficiently dynamic to modify the average values of the concentration and the vertical gradient of streamwise velocity. In other words, the layer decomposition is valid on average but not instantaneously at a given vertical position. Momentum and concentration can be transferred by a succession of frictional, collisional or turbulent mechanisms in a short time period. As this intermittency originates from large scale turbulence effects, it could explain why the local rheology and the Coulomb failure criterion fails in predicting the measurements.

\subsection{Turbulent fluid-particle interactions}

\section{Schmidt number modification}

The Schmidt number in sediment laden flows has been subject to many discussions in the literature over the past decades (e.g. Ismail 1951; Van Rijn 1984). Field and laboratory experiments showed that the Schmidt number value can substantially depart from unity (Graf \& Cellino 2002). Greimann et al. (1999) argued that the increased diffusion of large particles (Schmidt number lower than unity) originates from the added diffusive nature of the sediment's velocity fluctuations. Nielsen \& Teakle (2004) have argued that finite length effects in the vertical distributions of concentration and momentum can explain the decrease of the Schmidt number for increasing suspension number. The authors showed that the use of a first order Fickian diffusivity for the concentration is only valid if the characteristic length scale of the concentration distribution is much larger than the one of the momentum distribution. This hypothesis is valid for light particles and small suspension number $(S<<1)$ with an almost homogeneous distribution of the concentration over the water column. In such condition the vertical gradient of concentration is sufficiently low to neglect second order terms in the diffusivity model. However, for massive particles $(S>1)$ the vertical gradient of concentration can be very important in the bed region, inducing non negligible second order terms in the Fickian derivative approximation. Following Nielsen \& Teakle (2004)'s approach these higher order terms explain the reduction of the Schmidt number for massive particles. An important aspect is that the Schmidt number presented here is calculated from the measured momentum diffusivity which is significantly affected by the presence of particles. As a consequence, the Schmidt number modulation could be mainly induced by the reduction of the turbulence driven momentum-mixing efficiency. To the best of our knowledge a clear quantitative description of the physical processes responsible for the Schmidt number decrease with particles inertia has not yet been proposed.

\section{Turbulence modulation}

Villaret \& Trowbridge (1991) have suggested that the stratified flow analogy is applicable to turbulent sand suspension flows. However, their model fails to reproduce individual 
profiles and a dependency on the particle size has been observed. A stratified flow analogy cannot account for the particle size dependence. The authors concluded that the physical processes behind this dependence is unclear. The stratification analogy relies on the assumption that the sediment concentration acts as a passive scalar. Following Ferry \& Balachandar (2001), this assumption is fully satisfied when the particle size is smaller than the Kolmogorov dissipation scale. On the contrary, when the particles are massive and their diameter is larger than the Kolmogorov scale, typically of the order of the integral scale of turbulence, particle inertia can not be neglected and sediment concentration can no longer be considered as a passive scalar. In such conditions density stratification is insufficient to account for the complex interactions between the turbulent fluctuating motions of particles and elementary fluid parcels. In our conditions, the particle size is not negligible compared with the integral length scale of turbulence. This can be seen from the mixing length values shown in figure $8 \mathrm{c}$ considered as a proxy of the local integral scale of turbulence. The values are of the same order of magnitude as the particle diameter $d_{p}=3 \mathrm{~mm}$, supporting the invalidity of the passive scalar assumption due to particles inertia effects.

A better understanding of these complex fluid-particle turbulent interactions is investigated on the basis of particle-scale DNS simulations (e.g. Kidanemariam et al. 2013; Vowinckel et al. 2014) in conditions where the particles size is larger than the Kolmogorov length scale. However these approaches are still restricted to low bulk Reynolds numbers $O\left(10^{3}\right)$ compared to the present conditions of $O\left(10^{5}\right)$. To our knowledge, such numerical studies have never been applied so far to turbulent sheet-flow conditions. The combination of DNS and Large Eddy Simulations with small-scale datasets obtained from the present experimental configuration should improve our understanding of fluid-particle turbulent interactions in sediment transport problems. These high resolution experimental and numerical data will also provide guidelines for the improvement of transport equations in turbulence models.

\section{Conclusion}

A new high-resolution sheet flow dataset containing velocity, concentration, sediment flux and turbulent shear stress profiles has been presented in this paper. These measurements are used to discuss the validity of sheet flow properties and to assess different existing modelling approaches considering turbulent and granular processes.

The analysis of momentum and concentration diffusivities, mixing length, Stokes and Inertial numbers profiles confirms the flow decomposition into different layers proposed in the literature. Consistently with the mean velocity and concentration profiles, the flow can be divided into a dilute suspension layer $(\phi \lesssim 0.1)$ dominated by turbulent mechanisms and a dense bed layer $(\phi \gtrsim 0.1)$ dominated by granular mechanisms. The dense bed layer can be further divided into two sublayers, a dense frictional sublayer capped by a more dilute collisional sublayer.

In the suspension layer, a significant attenuation of the turbulent momentum diffusion efficiency is observed based on a significant reduction of the von Karman parameter compared to the clear water value. In this layer, the turbulent momentum diffusivity is shown to be proportional to the turbulent concentration diffusivity with an almost constant coefficient of proportionality equal to $\sigma_{s}=0.44$.

The mixing length in the suspension layer evolves linearly with $z$ as in the a clear water shear boundary layer but the origin of the linear mixing length profile is located in the bed layer, i.e. above the bed interface. Models are necessary for both the prediction of the von Karman parameter and the origin of the modified low of the wall in sheet 
flow conditions. Furthermore, the measurements showed that the turbulent momentum diffusion is affected by the solid transport in regions where the concentration and the stratification are negligible. This behaviour strongly supports the existence of non-local effects attributed to the presence of the sheet flow layer.

The validity of the Rouse model in the suspension layer, has been confirmed when all the required parameters are evaluated properly: the von Karman parameter, the Schmidt number, the shifted origin for the $z$ axis and the reference concentration value and location.

It has also been shown that the dense granular rheology cannot be applied straightforward to predict the measurements in the bed layer. Particularly the Coulomb failure criterion, which is commonly used in the models from the literature, is found to be much lower than the tangent of the measured angle of repose which is classically used in sheet-flow models. This might be explained by the bed layer intermittency attributed to the impact of large scale coherent flow structures. This large-scale-induced intermittency questions the relevance of a steady state local rheology and supports the need of including intermittency effects in sheet-flow models.

\section{Acknowledgments}

The authors acknowledge the DGA for the financial support of the first author PhD Thesis ( $\mathrm{N}^{\circ}$ 2011-170914/DGA/DS/MRIS). The development of the ACVP has been funded by the EU project Hydralab IV-WISE (contract 261520) and the experiments has been funded by the pole SMINGUE UJF/INPG and SEI-MEPIERA. The authors also gratefully acknowledge J.M. Barnoud for the technical support during the development of the experimental set-up. 


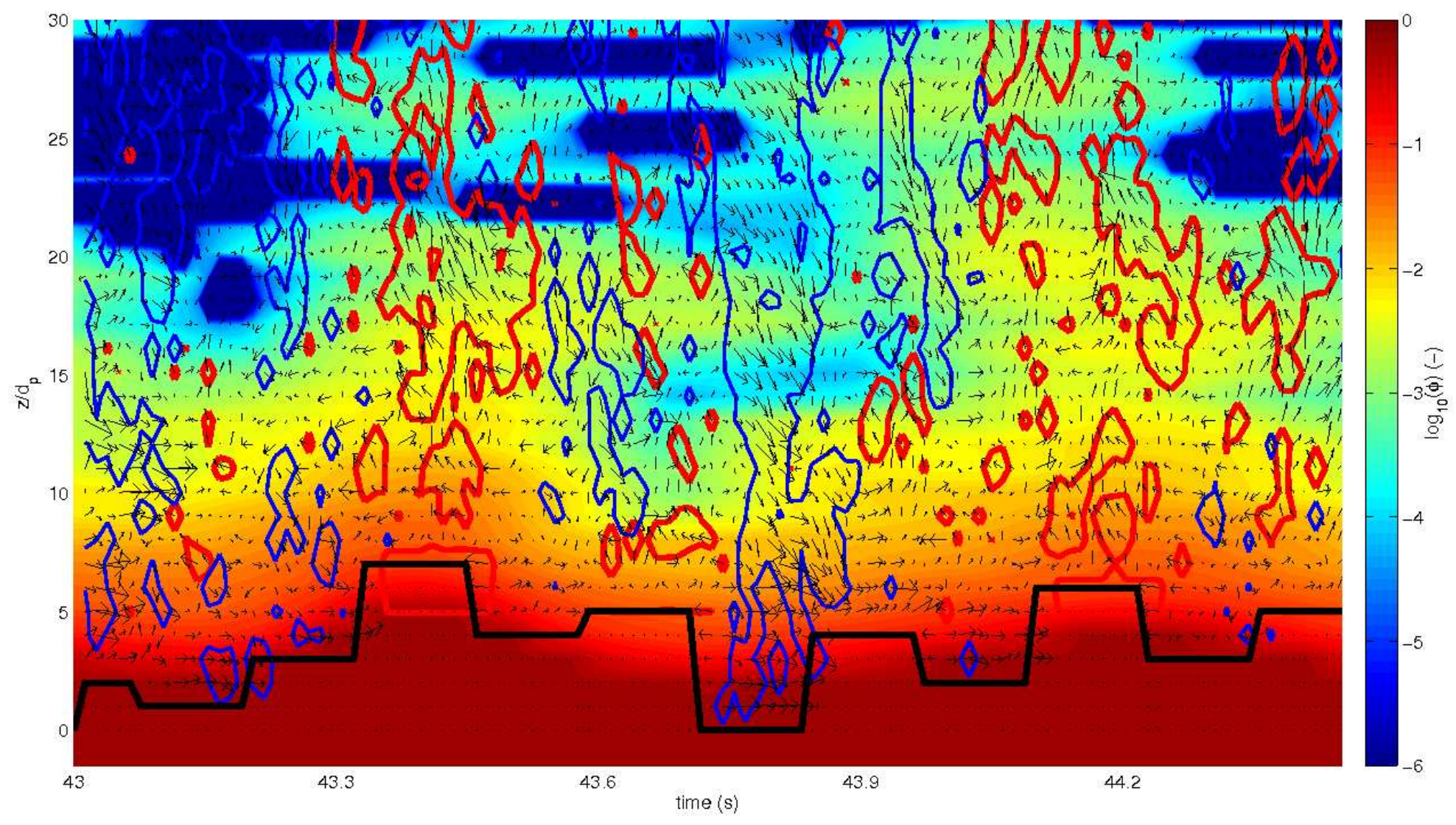

FIGURE 13. Time evolution of the measured bed interface position (black thick line) and concentration measurements (colorplot in base 10 logarithm) $£$ associated to coherent structures detection: the red and blue contours represent the ejections and sweeps respectively as iso-contours of $H=2$ as evaluated in Mignot et al. (2009). The vector plot represents the instantaneous $2 \mathrm{C}$ velocity fluctuations. 


\section{REFERENCES}

Amoudry, L., Hsu, T. J. \& Liu, P. L. F. 2008 Two-phase model for sand transport in sheet flow regime. J. Geophys. Res. 113.

Andreotti, B., Forterre, Y. \& Pouliquen, O. 2013 Granular Media: Between Fluid and Solid. Cambridge.

Armanini, A., Capart, H., Fraccarollo, L. \& Larcher, M. 2005 Rheological stratification in experimental free-surface flows of granular-liquid mixtures. Journal of Fluid Mechanics 532, 269-319.

Aussillous, P., Chauchat, J., Pailha, M., Médale, M. \& Guazzelli, E. 2013 Investigation of the mobile granular layer in bedload transport by laminar shearing flows. Journal of Fluid Mechanics 736, 594-615.

BAGNold, R. A. 1954 Experiments on a gravity-free dispersion of large solid spheres in a newtonian fluid under shear. Phil. Trans. R. Soc. Lond. 225, 49-63.

Bagnold, R. A. 1956 The flow of cohesionless grains in fluids. Phil. Trans. R. Soc. Lond. 249, 235-297.

Berzi, Diego 2011 Analytical solution of collisional sheet flows. Journal of Hydraulic Engineering 137 (10), 1200-1207.

Berzi, D. \& Fraccarollo, L. 2013 Inclined, collisional sediment transport. Physics of Fluids $25(10),-$.

Best, J., Bennett, S., Bridge, J. \& Leeder, M. 1997 Turbulence modulation and particle velocities over flat sand beds at low transport rates. Journal of Hydraulic Engineering 123 (12), 1118-1129.

Boyer, F., Guazzelli, E. \& Pouliquen, O. 2011 Unifying suspension and granular rheology. Phys. Rev. Lett. 107, 188301.

Capart, H. \& Fraccarollo, L. 2011 Transport layer structure in intense bed-load. Geophysical Research Letters 38 (20), n/a-n/a.

Cassar, C., Nicolas, M. \& Pouliquen, O. 2005 Submarine granular flows down inclined planes. Physics of Fluids 17 (10), 103301.

Castro-Orgaz, O., Giráldez, J. V., Mateos, L. \& Dey, S. 2012 Is the von kármán constant affected by sediment suspension? Journal of Geophysical Research: Earth Surface $\mathbf{1 1 7}$ (F4), $\mathrm{n} / \mathrm{a}-\mathrm{n} / \mathrm{a}$.

Chassagneux, F. X. \& Hurther, D. 2014 Wave bottom boundary layer processes below irregular surfzone breaking waves with light-weight sheet flow particle transport. Journal of Geophysical Research: Oceans 119 (3), 1668-1690.

Chauchat, J. \& Guillou, S. 2008 On turbulence closures for two-phase sediment-laden flows models. Journal Geophysical Research: Oceans 113, 20.

Chauchat, J. \& Médale, M. 2010 A 3D numerical model for incompressible two-phase flow of a granular bed submitted to a laminar shearing flow. Computer Methods in Applied Mechanics and Engineering 199, 439-449.

Chauchat, J. \& MÉdale, M. 2014 A three-dimensional numerical model for dense granular flows based on the rheology. Journal of Computational Physics 256 (0), 696-712.

Cowen, E. A., Dudley, R. D., Liao, Q., Variano, E. A. \& Liu, P. L.-F. 2010 An insitu borescopic quantitative imaging profiler for the measurement of high concentration sediment velocity. Experiments in Fluids 49 (1), 77-88.

DANIEL, SM 1965 Flow of suspension in a rectangular channel. PhD thesis.

Einstein, H. A. 1950 The bed load function for sedimentation in open channel channel flows. Tech. Rep. 1026. U.S. Department of Agriculture.

FERry, J. \& Balachandar, S. 2001 A fast eulerian method for disperse two-phase flow. International Journal of Multiphase Flow 27 (7), 1199-1226.

Forterre, Y. \& Pouliquen, O. 2008 Flows of dense granular media. Annual Review of Fluid Mechanics 40, 1-24.

Gaudio, R., Miglio, A. \& Dey, S. 2010 Non-universality of von kármán's in fluvial streams. Journal of Hydraulic Research 48 (5), 658-663.

GDR MIDI 2004 On dense granular flows. The European Physical Journal E 14, 341-365.

Graf, W. H. \& Cellino, M. 2002 Suspension flows in open channels; experimental study. Journal of Hydraulic Research 40, 435-447. 
Greimann, B. P., Muste, M. \& Jr., F. M. Holly 1999 Two-phase formulation of suspended sediment transport. Journal of Hydraulique Research 37, 479 - 500.

Hanes, Daniel M. \& Inman, Douglas L. 1985 Experimental evaluation of a dynamic yield criterion for granular fluid flows. Journal of Geophysical Research: Solid Earth 90 (B5), 3670-3674.

Hsu, T.J., Jenkins, J. T. \& LiU, P. L.-F. 2004 On two-phase sediment transport: sheet flow of massive particles. Proceedings of the Royal Society of London. Series A: Mathematical, Physical and Engineering Sciences 460 (2048), 2223-2250.

Hurther, D. \& Lemmin, U. 2001 Shear stress statistics and wall similarity analysis in turbulent boundary layers using a high resolution 3d advp. Journal of Oceanic Engineering 25, 446457.

Hurther, D. \& Lemmin, U. 2008 Improved turbulence profiling with field-adapted acoustic doppler velocimeters using a bifrequency doppler noise suppression method. Journal of Atmospheric and Oceanic Technology 25, 452-463.

Hurther, D., Lemmin, U. \& Terray, E. A. 2007 Turbulent transport in the outer region of rough-wall open-channel flows: the contribution of large coherent shear stress structures (lc3s). Journal of Fluid Mechanics 574, 465-493.

Hurther, D. \& Thorne, Peter D. 2011 Suspension and near-bed load sediment transport processes above a migrating, sand-rippled bed under shoaling waves. Journal of Geophysical Research: Oceans 116 (C7), n/a-n/a.

Hurther, D., Thorne, P. D., Bricault, M., Lemmin, U. \& Barnoud, J.M. 2011 A multifrequency acoustic concentration and velocity profiler (acvp) for boundary layer measurements of fine-scale flow and sediment transport processes. Coastal Engineering 58, 594-605.

Ismail, H.M. 1951 Turbulent transfer mechanism and suspended sediment in closed channels. Proc. ASCE 77(6).

Jenkins, J. T. 2007 Dense inclined flow of inelastic spheres. Granular matter 10, 47-52.

Jenkins, J. T. \& HANES, D. M. 1998 Collisional sheet flows of sediment driven by a turbulent fluid. Journal of Fluid Mechanics 370 (-1), 29-52.

Kidanemariam, A.G. Chan-Braun, C. Doychev, T. \& Uhlmann, M. 2013 Direct numerical simulation of horizontal open channel flow with finite-size, heavy particles at low solid volume fraction. New J. Phys. 15 (2), 025031.

Lu, S. S. \& Willmarth, W. W. 1973 Measurements of the structure of the reynolds stress in a turbulent boundary layer. Journal of Fluid Mechanics 60, 481-511.

Meyer-Peter, E. \& Muller, R. 1948 Formulas for bed-load transport. In 2nd Meeting of the International Association of Hydraulic and Structural Research, pp. 34-64.

Mignot, E., Hurther, D. \& Barthelemy, E. 2009 On the structure of shear stress and turbulent kinetic energy flux across the roughness layer of a gravel-bed channel flow. Journal of Fluid Mechanics 638, 423-452.

Muste, M., Yu, K., Fujita, I. \& Ettema, R. 2005 Two-phase versus mixed-flow perspective on suspended sediment transport in turbulent channel flows. Water Resour Res 41, 22.

Muste, M., Yu, K. \& Spasojevic, M. 2004 Practical aspects of adcp data use for quantification of mean river flow characteristics; part i: moving-vessel measurements. Flow Measurement and Instrumentation 15 (1), 1-16.

Naqshband, S., Ribberink, J. S., Hurther, D. \& Hulscher, S.J.M.H. 2014 Bed load and suspended load contributions to migrating sand dunes in equilibrium. Journal of Geophysical Research: Earth Surface pp. n/a-n/a.

Nielsen, P. \& Teakle, I. A. L. 2004 Turbulent diffusion of momentum and suspended particles: A finite-mixing-length theory. Physics of Fluids 16 (7), 2342-2348.

NnAdi, F. N. \& Wilson, K. C. 1992 Motion of contact-load particles at high shear stress. Journal of Hydraulic Engineering 118 (12), 1670-1684.

Ouriemi, M., Aussillous, P. \& Guazzelli, E. 2009 Sediment dynamics. Part I: Bed-load transport by shearing flows. Journal of Fluid Mechanics 636, 295-319.

Pasini, J. M. \& Jenkins, J. T. 2005 Aeolian transport with collisional suspension. Philosophical Transactions of the Royal Society A: Mathematical, Physical and Engineering Sciences 363 (1832), 1625-1646.

Prandtl, L. 1926 Bericht über neuere turbulenzforschung. Hydraulische Probleme pp. 1-13. 
Pugh, F. J. \& Wilson, K. C. 1999 Velocity and concentration distributions in sheet flow above plane beds. Journal of Hydraulic Engineering 125 (2), 117-125.

Revil-Baudard, T. \& Chauchat, J. 2013 A two-phase model for sheet flow regime based on dense granular flow rheology. Journal of Geophysical Research: Oceans 118 (2), 619-634.

Rouse, H. 1937 Modern conceptions of the mechanics of turbulence. Trans. Am. Soc. Civ. Eng. 102, $463-505$.

Song, T. \& Graf, W. H. 1994 Non uniform open-channel flow over a rough bed. Journal of Hydroscience and Hydraulic Engineering 12 (1), 1-25.

Spinewine, B., Capart, H., Fraccarollo, L. \& Larcher, M. 2011 Laser stripe measurements of near-wall solid fraction in channel flows of liquid-granular mixtures. Experiments in Fluids 50, 1507-1525.

Sumer, B. M., Kozakiewicz, A., Fredsøe, J. \& Deigaard, R. 1996 Velocity and concentration profiles in sheet-flow layer of movable bed. Journal of Hydraulic Engineering 122 (10), 549-558.

Thorne, P. D. \& Hanes, D. M. 2002 A review of acoustic measurement of small-scale sediment processes. Continental Shelf Research 22 (4), 603-632.

Thorne, P. D. \& Hurther, D. 2014 An overview on the use of backscattered sound for measuring suspended particle size and concentration profiles in non-cohesive inorganic sediment transport studies. Continental Shelf Research 73 (0), 97-118.

Thorne, P. D., Hurther, D. \& Moate, B. D. 2011 Acoustic inversions for measuring boundary layer suspended sediment processes. The Journal of the Acoustical Society of America 130 (3), 1188-1200.

van Der A, D., ODonoghue, T., Davies, A. G. \& Ribberink, J. S. 2011 Experimental study of the turbulent boundary layer in acceleration-skewed oscillatory flow. Journal of Fluid Mechanics 684, 251-283.

Van Rijn, L. C. 1984 Sediment transport, part ii: Suspended load transport. J. Hydraul. Eng. 110, 1613-1641.

VAnoni, V. A. 1975 Sedimentation engineering. New York: American Society of Civil Engineers.

VANONI, V. A. \& BRooks, N. H. 1957 Laboratory studies of the roughness and suspended load of alluvial streams. Sedimentation Laboratory, California Institute of Technology, Pasadena, Calif.

Villaret, C. \& Trowbridge, J. H. 1991 Effects of stratification by suspended sediments on turbulent shear flows. Journal of Geophysical Research: Oceans 96 (C6), 10659-10680.

Vowinckel, B., Kempe, T. \& Fröhlich, J. 2014 Fluid-particle interaction in turbulent open channel flow with fully-resolved mobile beds. Advances in Water Resources 72 (0), 32-44, \{THESIS\} (Two-pHase modElling for Sediment dynamIcS).

Wilson, Kenneth C. 1966 Bed-load transport at high shear stress. In Proc. A.S.C.E, , vol. HY6. ASCE.

Wilson, K. C. 1989 Mobile-bed friction at high shear stress. Journal of Hydraulic Engineering 115 (6), 825-830.

YALin, M. S. 1977 Mechanics of sediment transport 2nd edition. Pergamon Press.

YANG, S.-Q. \& CHOw, A. T. 2008 Turbulence structures in non-uniform flows. Advances in Water Resources 31 (10), 1344-1351. 